\title{
CR WEYL GEOMETRY AND CONNECTIONS OF THE CANONICAL BUNDLE
}

\author{
TAKAAKI OHKUBO
}

\begin{abstract}
A nondegenerate $\mathrm{CR}$ manifold has a natural conformal class consisting of hermitian forms, which are called Levi forms, on the hyperdistribution. It is important to study the objects on CR manifolds which are independent of the choice of Levi forms. A CR Einstein-Weyl structure defined in [8] is one of such objects. Our purpose is to find nondegenerate CR manifolds with CR Einstein-Weyl structures.

In this paper, we shall obtain many examples of CR Einstein-Weyl manifolds. These examples are actually derived from the fact that CR Einstein-Weyl structures are closely concerned with connections of the canonical bundle of CR manifolds.
\end{abstract}

\section{Introduction}

We have been considering the following problem: Find nondegenerate CR manifolds with CR Einstein-Weyl structures. Actually we have the only one example in [8] of CR Einstein-Weyl manifolds.

A nondegenerate $\mathrm{CR}$ manifold has a natural conformal class consisting of hermitian forms, which are called Levi forms, on the hyperdistribution. Accordingly the geometry on a CR manifold has been compared with that on a usual conformal manifold with a conformal class consisting of Riemannian metrics. The objects which are invariant under the conformal change of Riemannian metrics are important. Correspondingly it is natural to consider that the objects which are independent of the choice of Levi forms have significance for CR geometry. A CR Einstein-Weyl structure appearing in the above problem is one of such objects on CR manifolds. Therefore it is consequential in CR geometry to study the various problems with respect to CR Einstein-Weyl structures.

A CR Einstein-Weyl structure on a nondegenerate CR manifold is introduced in [8] as an analogous notion to an Einstein-Weyl structure.

The Einstein-Weyl structure is one of the above mentioned objects in conformal geometry and, in fact, is a pair of a conformal class of Riemannian

2000 Mathematics Subject Classification. Primary 53c25; Secondary 53c26.

Received March 18, 2005; revised July 27, 2005. 
metrics and a torsion-free linear connection preserving the conformal class (Weyl connection) whose Ricci tensor satisfies the equation that the symmetric part is proportional to the Riemannian metrics belonging to the conformal class pointwise. We can say that the Einstein-Weyl structure is the conformal version of the Einstein structure.

The CR Einstein-Weyl structure is similarly defined for the conformal class of Levi forms on nondegenerate CR manifolds, but the definition has a certain remarkable point. This point is that the CR Einstein-Weyl structure is the pseudoconformal version of the pseudo-Einstein structure.

The pseudo-Einstein structure is the notion introduced by J. M. Lee in [7] and correponds to the Einstein structure. In fact, the pseudo-Einstein structure is as follows: On a nondegenerate $\mathrm{CR}$ manifold, there is a class consisting of particular almost contact structures belonging to the CR structure and the Levi forms are actually induced by the almost contact structures. Therefore when we compare CR geometry with conformal geometry, we had better consider that the almost contact structures correspond to the Riemannian structures. Fixed an almost contact structure belonging to the $\mathrm{CR}$ structure, there is a unique linear connection associated with the almost contact structure which is called the Tanaka-Webster connection. It follows that Tanaka-Webster connections correspond to Levi-Civita connections. An almost contact structure is pseudoEinstein if the $*$-Ricci tensor of the Tanaka-Webster connection is proportional to the Levi form pointwise. Here, the $*$-Ricci tensor is the hermitian tensor field defined in [10] and is more natural in CR geometry than the usual Ricci tensor.

In fact, the linear connection corresponding to the Weyl connection in the definition of CR Einstein-Weyl structures (CR Weyl connection) is defined as the connection which is preserving the class of the almost contact structures and is closely concerned with Tanaka-Webster connections. A CR Weyl connection is CR Einstein-Weyl if the hermitian part of the $*$-Ricci tensor of the CR Weyl connection is proportional to the Levi forms pointwise.

Seemingly the pseudo-Einstein structure is fundamental, but it depends on the choice of the Levi forms. Therefore the author considers that we should attach importance to CR Einstein-Weyl structures rather than pseudo-Einstein structures. However we should keep it in mind that CR Einstein-Weyl structures is closely related to pseudo-Einstein structures.

In this paper, we shall show the following theorem (Theorem 5.1): Let $N$ be a complex manifold with a holomorphic connection of the canonical line bundle. Then every nondegenerate real hypersurface in $N$ admits a CR EinsteinWeyl structure. Theorem 5.1 provides us with many examples of CR EinsteinWeyl manifolds. Concretely we can see these in Examples 1, 2 and 3 in Section 5. For instance, we obtain the following corollary (Corollary 5.3) in Example 3: Let $N$ be a compact Kählerian manifold with zero first Chern class. Then every nondegenerate real hypersurface in $N$ admits a CR Einstein-Weyl structure.

Theorem 5.1 is brought about by the result which Lee obtained in [7]. In fact, it is as follows: If there is a nonvanishing closed $(n+1,0)$-form on an orientable nondegenerate $\mathrm{CR}$ manifold $M$ of dimension $2 n+1(n \geq 1), \quad M$ 
admits a pseudo-Einstein structure. This Lee's work becomes a great help to obtain the key theorem (Theorem 3.3) which induces Theorem 5.1. Theorem 3.3 states that connections of the canonical bundle on CR manifolds are closely concerned with CR Einstein-Weyl structures.

The canonical bundle on CR manifolds is appeared in various studies [2] and [3]. CR Weyl connections and CR Einstein-Weyl structures would be useful for the study of CR geometry through connections of the canonical bundle.

In Sections 1 and 2, we recall the definitions of Tanaka-Webster connections, pseudo-Einstein structures, CR Weyl connections and CR Einstein-Weyl structures. In Section 3, we give a new proof of the above theorem which Lee obtained in [7]. This viewpoint makes us think of Theorem 3.3. In fact, Theorem 3.3 gives a sufficient condition for the existence of CR Einstein-Weyl structures on orientable CR manifolds. In Section 4, we observe that Theorem 3.3 holds even if CR manifolds are not necessarily orientable. Moreover we show in Proposition 4.3 that the assumtion of Theorem 3.3 is the necessary condition for the existence of CR Einstein-Weyl structures. The last section is devoted to Theorem 5.1 and the examples of CR Einstein-Weyl manifolds.

\section{Tanaka-Webster connections}

Let $M$ be a connected differentiable manifold of dimention $2 n+1(n \geq 1)$. Let $\mathscr{D}$ denote a 1 -codimensional subbundle of the tangent bundle $T M$, which is called a hyperdistribution. A cross section $J$ of the bundle $\mathscr{D} \otimes \mathscr{D}^{*}$ satisfying $J^{2}=-I$ is called a complex structure on $\mathscr{D}$, where $\mathscr{D}^{*}$ is the dual bundle of $\mathscr{D}$ and $I$ is the identity transformation. Let $(\mathscr{D}, J)$ be a pair of a hyperdistribution $\mathscr{D}$ and a complex structure $J$ on $\mathscr{D}$. The complex structure $J$ on $\mathscr{D}$ can be uniquely extended to a complex linear endomorphism of the complexification $\mathbf{C} \mathscr{D}$ of $\mathscr{D}$ and the extended endomorphism, denoted also by $J$, satisfies the equation $J^{2}=-I$. Thus the vector bundle $\mathbf{C} \mathscr{D}$ is decomposed as

$$
\mathbf{C} \mathscr{D}=\mathscr{D}^{1,0} \oplus \mathscr{D}^{0,1} \text {, }
$$

where $\mathscr{D}^{1,0}$ (resp. $\mathscr{D}^{0,1}$ ) be a subbundle of $\mathbf{C} \mathscr{D}$ composed of the eigenvectors corresponding to $i$ (resp. $-i$ ) of $J$. A pair $(\mathscr{D}, J)$ is a $C R$ structure if $\mathscr{D}^{1,0}$ is involutive, that is, $\mathscr{D}^{1,0}$ satisfies

$$
\left[Y_{1}, Y_{2}\right] \in \Gamma\left(\mathscr{D}^{1,0}\right)
$$

for any $Y_{1}, Y_{2} \in \Gamma\left(\mathscr{D}^{1,0}\right)$, where $\Gamma\left(\mathscr{D}^{1,0}\right)$ denotes the set of all cross sections of the vector bundle $\mathscr{D}^{1,0}$. A pair $(\mathscr{D}, J)$ is a CR structure if and only if the following two conditions hold:

$$
\begin{gathered}
{[J X, J Y]-[X, Y] \in \Gamma(\mathscr{D})} \\
{[J X, J Y]-[X, Y]-J([X, J Y]+[J X, Y])=0}
\end{gathered}
$$

for any $X, Y \in \Gamma(\mathscr{D})$ (cf. [9]). If $M$ admits a CR structure $(\mathscr{D}, J)$, then $(M, \mathscr{D}, J)$ is called a $C R$ manifold. Let $\theta$ be a local 1 -form on an open set $U \subset M$ 
annihilating the hyperdistribution $\mathscr{D}$, which is determined up to nonvanishing smooth functions. Noting that

$$
-2 d \theta(X, Y)=\theta([X, Y])
$$

for any $X, Y \in \Gamma(U, \mathscr{D})$, we see that the condition (C.1) for the pair $(\mathscr{D}, J)$ is equivalent to the condition

$$
d \theta(J X, J Y)=d \theta(X, Y)
$$

for all $X, Y \in \mathscr{D}$, where $\Gamma(U, \mathscr{D})$ denotes the set of all cross sections on $U$ of the vector bundle $\mathscr{D}$. The base space $M$ of the vector bundle $\mathscr{D}$ may be restricted on an open set according to the objects of $\mathscr{D}$. Moreover we have

$$
d(f \theta)(X, Y)=f d \theta(X, Y)
$$

for all $X, Y \in \mathscr{D}$ and every smooth function $f$ on $U$, which allows us to call $(\mathscr{D}, J)$ a nondegenerate CR structure if $d \theta$ is nondegenerate on $\mathscr{D}$.

Let $(M, \mathscr{D}, J)$ be a nondegenerate $\mathrm{CR}$ manifold and $\theta$ a local 1 -form on an open set $U$ annihilating $\mathscr{D}$. For $\theta$, there uniquely exists $\xi \in \Gamma(T U)$ satisfying

$$
\begin{aligned}
\theta(\xi) & =1, \\
{[\xi, \Gamma(U, \mathscr{D})] } & \subset \Gamma(U, \mathscr{D})
\end{aligned}
$$

(cf. [9]). Then defining the tensor field $\phi$ on $U$ by

$$
\phi(V)=J(V-\theta(V) \xi)
$$

for every $V \in T U$, the triplet $(\phi, \xi, \theta)$ becomes an almost contact structure on $U$ (cf. [9]). Such an almost contact structure $(\phi, \xi, \theta)$ satisfying the condition $(*)$ is called a $\mathscr{D}$-preserving almost contact structure belonging to $(\mathscr{D}, J)$ and is written as $(U,(\phi, \xi, \theta))$ (cf. [8]).

Remark. Note that if $M$ is orientable, there exist $\mathscr{D}$-preserving almost contact structures $(\phi, \xi, \theta)$ globally defined on $M$. Fix an orientation $\mathcal{O}$ on $M$. Usually assumed that a manifold is second countable, there is a Riemannian metric $h$ on $M$. We take a base $\left\{e_{1}, \ldots, e_{n}, J e_{1}, \ldots, J e_{n}\right\}$ of $\mathscr{D}$ at each point $x \in M$. Then we can take a differentiable unit vector field $\xi^{\circ}$ such that it is orthogonal to $\mathscr{D}$ with respect to $h$ and $\left\{\xi^{\circ}, e_{1}, \ldots, e_{n}, J e_{1}, \ldots, J e_{n}\right\}$ is a positive base of the orientation $\mathcal{O}$. We put $\theta(V)=h\left(\xi^{\circ}, V\right)$ and $\phi^{\circ}(V)=J\left(V-\theta(V) \xi^{\circ}\right)$ for every $V \in T M$. Then $\left(\phi^{\circ}, \xi^{\circ}, \theta\right)$ becomes an almost contact structure on $M$. This almost contact structure may not satisfy the condition $(*)$. However by Proposition 1.2 in [9], we can always obtain a $\mathscr{D}$-preserving almost contact structure globally defined on $M$. $\omega$ by

Let $(U,(\phi, \xi, \theta))$ be a $\mathscr{D}$-preserving almost contact structure on $U$. Define

$$
\omega=-2 d \theta
$$


Moreover define $g: \mathscr{D} \times \mathscr{D} \rightarrow \mathbf{R}$ by

$$
g(X, Y)=\omega(J X, Y),
$$

which satisfies the equations

$$
g(X, Y)=g(Y, X), \quad g(J X, J Y)=g(X, Y)
$$

for all $X, Y \in \mathscr{D}$, where we have used (1.2). Therefore $g$ is hermitian and nondegenerate on $\mathscr{D}$ and is called Levi form with respect to $(U,(\phi, \xi, \theta))$. On nondegenerate $\mathrm{CR}$ manifold $(M, \mathscr{D}, J)$, Tanaka [12] showed (cf. [9]):

LemMa 1.1. Let $(U,(\phi, \xi, \theta))$ be a D-preserving almost contact structure on $U$. Then there uniquely exists a linear connection $\nabla$ on the open submanifold $U$ such that

$$
\begin{gathered}
\nabla \phi=0, \quad \nabla \xi=0, \quad \nabla \theta=0, \quad \nabla^{\circ} g=0, \\
T^{\nabla}(X, Y)=-\omega(X, Y) \xi, \quad T^{\nabla}(\xi, Y)=-\frac{1}{2} \phi\left(\mathscr{L}_{\xi} \phi\right) Y
\end{gathered}
$$

for all $X, Y \in \mathscr{D}$, where $\nabla^{\circ}$ denotes the induced connection on the hyperdistribution $\mathscr{D}, T^{\nabla}$ the torsion tensor of $\nabla$ and $\mathscr{L}_{\xi}$ the Lie differentiation with respect to $\xi$.

The linear connection in the above lemma is called the Tanaka-Webster connection associated with $(U,(\phi, \xi, \theta))$.

For a $\mathscr{D}$-preserving almost contact structure $(U,(\phi, \xi, \theta))$, we can take a frame field of CTU:

$$
\left\{\xi, Z_{1}, \ldots, Z_{n}, Z_{\overline{1}}, \ldots, Z_{\bar{n}}\right\}
$$

where we shrink $U$ if necessary, $\left\{Z_{1}, \ldots, Z_{n}\right\}$ is a frame field of $\mathscr{D}^{1,0}$ on $U$ and $Z_{\bar{\alpha}}=\overline{Z_{\alpha}}$. We call such a frame field containing $\xi$ an admissible frame of $(U,(\phi, \xi, \theta))$. We put the dual frame field of (1.6) as

$$
\left\{\theta, \theta^{1}, \ldots, \theta^{n}, \theta^{\overline{1}}, \ldots, \theta^{\bar{n}}\right\},
$$

where $\theta^{\bar{\alpha}}=\overline{\theta^{\alpha}}$. The set $\left\{\theta^{1}, \ldots, \theta^{n}\right\}$ in (1.7) is called the admissible coframe with respect to $\theta$ (cf. [6]).

Remark. We uniquely extend $g$ to a complex bilinear form of $\mathbf{C} \mathscr{D}$, denoted also by $g$, and we put

$$
g_{\alpha \bar{\beta}}=g\left(Z_{\alpha}, Z_{\bar{\beta}}\right), \quad g_{\bar{\alpha} \beta}=g\left(Z_{\bar{\alpha}}, Z_{\beta}\right)
$$

for the adapted frame (1.6). From the property (1.5) of $g$, it follows that the matrices $\left(g_{\alpha \bar{\beta}}\right)$ and $\left(g_{\bar{\alpha} \beta}\right)$ satisfy the equations $g_{\alpha \bar{\beta}}=g_{\bar{\beta} \alpha}$ and $\overline{g_{\alpha \bar{\beta}}}=g_{\bar{\alpha} \beta}$, and moreover these are the nondegenerate matrices. We put

$$
\left(g^{\bar{\alpha} \beta}\right)=\left(g_{\alpha \bar{\beta}}\right)^{-1}, \quad\left(g^{\alpha \bar{\beta}}\right)=\left(g_{\bar{\alpha} \beta}\right)^{-1},
$$


where $\left(g_{\alpha \bar{\beta}}\right)^{-1}$ (resp. $\left.\left(g_{\bar{\alpha} \beta}\right)^{-1}\right)$ denotes the inverse matrix of $\left(g_{\alpha \bar{\beta}}\right)$ (resp. $\left.\left(g_{\bar{\alpha} \beta}\right)\right)$. Then we have $g^{\alpha \bar{\beta}}=g^{\bar{\beta} \alpha}$ and $g^{\alpha \bar{\beta}}=g^{\bar{\alpha} \beta}$. In the sequel small Greek indices run from 1 to $n$, and Einstein convention is used. The Levi form $g_{\alpha \bar{\beta}}$ and its inverse $g^{\bar{\alpha} \beta}$ are used to lower and raise indices. On the other hand, note that $d \theta$ is written as

$$
d \theta=i g_{\alpha \bar{\beta}} \theta^{\alpha} \wedge \theta^{\bar{\beta}}
$$

in virtue of $(*)$.

We uniquely extend the covariant derivative of the Tanaka-Webster connection associated with $(U,(\phi, \xi, \theta))$ to the covariant derivative of $\mathbf{C} T U$ and it is also denoted by $\nabla$. When we write the connection form with respect to (1.6) as

$$
\left(\begin{array}{ccc}
0 & & \\
& v_{\beta}^{\gamma} & \\
& & v_{\bar{\beta}}^{\bar{\gamma}}
\end{array}\right),
$$

we know that (1.9) satisfies

$$
\begin{gathered}
d \theta^{\gamma}+v_{\beta}^{\gamma} \wedge \theta^{\beta}=-A_{\bar{\beta}}^{\gamma} \theta^{\bar{\beta}} \wedge \theta, \quad A_{\alpha \beta}=A_{\beta \alpha}, \\
v_{\alpha \bar{\beta}}+v_{\bar{\beta} \alpha}=d g_{\alpha \bar{\beta}},
\end{gathered}
$$

where $v_{\bar{\beta}}^{\bar{\gamma}}=\overline{v_{\beta}{ }^{\gamma}}, A_{\beta}^{\bar{\gamma}}=\overline{A_{\bar{\beta}}^{\gamma}}, A_{\alpha \beta}=g_{\alpha \bar{\gamma}} A_{\beta}^{\bar{\gamma}_{\beta}}$ and $v_{\alpha \bar{\beta}}=v_{\alpha}^{\gamma} g_{\gamma \bar{\beta}}$ (cf. [6], [11], [13]).

Taking any two $\mathscr{D}$-preserving almost contact structures $(U,(\phi, \xi, \theta))$ and $\left(U^{\prime},\left(\phi^{\prime}, \xi^{\prime}, \theta^{\prime}\right)\right)$ such that $U \cap U^{\prime} \neq \emptyset$, these are related as follows (cf. [9]):

LEMMA 1.2. There is a unique smooth function $\mu$ on $U \cap U^{\prime}$ such that

$$
\theta^{\prime}=\varepsilon e^{2 \mu} \theta, \quad \xi^{\prime}=\varepsilon e^{-2 \mu}\left(\xi-2 Q^{*}\right), \quad \phi^{\prime}=\phi-2 \theta \otimes P^{*}, \quad g^{\prime}=\varepsilon e^{2 \mu} g,
$$

where $\varepsilon= \pm 1, \quad P^{*} \in \Gamma\left(U \cap U^{\prime}, \mathscr{D}\right)$ is defined by $g\left(P^{*}, X\right)=d \mu(X)$ for every $X \in \Gamma\left(U \cap U^{\prime}, \mathscr{D}\right)$ and $Q^{*}=J P^{*}$.

Let $\nabla$ and $\nabla^{\prime}$ be the Tanaka-Webster connections associated with $(U,(\phi, \xi, \theta))$ and $\left(U^{\prime},\left(\phi^{\prime}, \xi^{\prime}, \theta^{\prime}\right)\right)$ respectively. Then it is known in [9] that

Lemma 1.3. Define the difference $H^{*}$ between $\nabla$ and $\nabla^{\prime}$ by $H^{*}=\nabla^{\prime}-\nabla$. Then on $U \cap U^{\prime}$, we have

$$
\begin{aligned}
H^{*}(X, Y)= & p^{*}(X) Y+p^{*}(Y) X-g(X, Y) P^{*} \\
& +q^{*}(X) J Y+q^{*}(Y) J X-g(J X, Y) Q^{*},
\end{aligned}
$$

for all $X, Y \in \Gamma\left(U \cap U^{\prime}, \mathscr{D}\right)$, where $p^{*}=d \mu$ and $q^{*}=-p^{*} \circ \phi$.

We write the expression of $d \mu$ with respect to (1.7) as 


$$
d \mu=\mu_{\alpha} \theta^{\alpha}+\mu_{\bar{\alpha}} \theta^{\bar{\alpha}}+\mu_{0} \theta .
$$

Then we have

(1.16) $\quad q^{*}=-i\left(\mu_{\alpha} \theta^{\alpha}-\mu_{\bar{\alpha}} \theta^{\bar{\alpha}}\right), \quad P^{*}=\mu^{\alpha} Z_{\alpha}+\mu^{\bar{\alpha}} Z_{\bar{\alpha}}, \quad Q^{*}=i\left(\mu^{\alpha} Z_{\alpha}-\mu^{\bar{\alpha}} Z_{\bar{\alpha}}\right)$, where $\mu^{\alpha}=\mu_{\bar{\beta}} g^{\bar{\beta} \alpha}$ and $\mu^{\bar{\alpha}}=\mu_{\beta} g^{\beta \bar{\alpha}}$.

Let

$$
\left\{\xi^{\prime}, Z_{1}^{\prime}, \ldots, Z_{n}^{\prime}, Z_{\overline{1}}^{\prime}, \ldots, Z_{\bar{n}}^{\prime}\right\}
$$

be an admissible frame of $\left(U^{\prime},\left(\phi^{\prime}, \xi^{\prime}, \theta^{\prime}\right)\right)$ such that $Z_{\alpha}^{\prime}=Z_{\alpha}$ on $U \cap U^{\prime}$. The relation between (1.7) and the admissible coframe

$$
\left\{\theta^{\prime},{ }^{\prime} \theta^{1}, \ldots,{ }^{\prime} \theta^{n},{ }^{\prime} \theta^{\overline{1}}, \ldots,{ }^{\prime} \theta^{\bar{n}}\right\},
$$

is

$$
{ }^{\prime} \theta^{\alpha}=\theta^{\alpha}+2 i \mu^{\alpha} \theta .
$$

We write the connection form of the Tanaka-Webster connection $\nabla^{\prime}$ associated with $\left(U^{\prime},\left(\phi^{\prime}, \xi^{\prime}, \theta^{\prime}\right)\right)$ with respect to the admissible frame (1.17) as

$$
\left(\begin{array}{lll}
0 & & \\
& { }^{\prime} v_{\beta} \gamma & \\
& & { }^{-\bar{\gamma}} \bar{\beta}^{-}
\end{array}\right) .
$$

We prepare notations before stating the following lemma. In general, expressing a 1 -form $p$ as

$$
p=p_{\alpha} \theta^{\alpha}+p_{\bar{\alpha}} \theta^{\bar{\alpha}}+p_{0} \theta
$$

on $U$, the notation $p_{\beta \lambda}$ (resp. $p_{\bar{\beta} \lambda}$ ) denotes the coefficient of $\theta^{\beta} \otimes \theta^{\lambda}$ (resp. $\left.\theta^{\bar{\beta}} \otimes \theta^{\lambda}\right)$ of $\nabla p$, where $(\nabla p)(V, W)=\left(\nabla_{W} p\right)(V)$ for $V, W \in T U$, namely,

$$
p_{\beta \lambda}=Z_{\lambda} \cdot p_{\beta}-v_{\beta}{ }^{\alpha}\left(Z_{\lambda}\right) p_{\alpha}, \quad p_{\bar{\beta} \lambda}=Z_{\lambda} \cdot p_{\bar{\beta}}-v_{\bar{\beta}}{ }^{\bar{\alpha}}\left(Z_{\lambda}\right) p_{\bar{\alpha}} .
$$

We similarly define $p_{\beta \bar{\lambda}}, p_{\bar{\beta} \bar{\lambda}}, p_{\beta 0}$ and $p_{\bar{\beta} 0}$. Note that

$$
p_{0 \lambda}=Z_{\lambda} \cdot p_{0}, \quad p_{0 \bar{\lambda}}=Z_{\bar{\lambda}} \cdot p_{0}, \quad p_{00}=\xi \cdot p_{0} .
$$

Define $P \in \Gamma(U, \mathscr{D})$ by $g(P, X)=p(X)$ for every $X \in \Gamma(U, \mathscr{D})$. We easily obtain that the expression of $P$ with respect to $(1.6)$ is

$$
P=p^{\alpha} Z_{\alpha}+p^{\bar{\alpha}} Z_{\bar{\alpha}},
$$

where $p^{\alpha}=g^{\alpha \bar{\beta}} p_{\bar{\beta}}$ and $p^{\bar{\alpha}}=g^{\bar{\alpha} \beta} p_{\beta}$. The notation $p_{\lambda}^{\beta}$ (resp. $p_{\lambda}^{\bar{\beta}}{ }_{\lambda}$ ) denotes the coefficient of $Z_{\beta} \otimes \theta^{\lambda}$ (resp. $\left.Z_{\bar{\beta}} \otimes \theta^{\lambda}\right)$ of $\nabla P$, that is,

$$
p_{\lambda}^{\beta}=Z_{\lambda} \cdot p^{\beta}+v_{\alpha}^{\beta}\left(Z_{\lambda}\right) p^{\alpha}, \quad p_{\lambda}^{\bar{\beta}}=Z_{\lambda} \cdot p^{\bar{\beta}}+v_{\bar{\alpha}}^{\bar{\beta}}\left(Z_{\lambda}\right) p^{\bar{\alpha}} .
$$

We similarly define $p^{\beta}{ }_{\bar{\lambda}}, p^{\bar{\beta}}{ }_{\bar{\lambda}}, p^{\beta}{ }_{0}$ and $p^{\bar{\beta}}{ }_{0}$. From (1.11), we have

$$
v^{\alpha \bar{\beta}}+v^{\bar{\beta} \alpha}=-d g^{\alpha \bar{\beta}} .
$$


By this result, we obtain

$$
p_{\lambda}^{\beta}=g^{\beta \bar{\alpha}} p_{\bar{\alpha} \lambda} .
$$

We have the similar results for $p^{\bar{\beta}}{ }_{\lambda}, p^{\beta}{ }_{\bar{\lambda}}, p^{\bar{\beta}}{ }_{\bar{\lambda}}, p^{\beta}{ }_{0}$ and $p^{\bar{\beta}}{ }_{0}$, that is, the covariant derivative with respect to the Tanaka-Webster connection is commutative with the lowering and raising indices.

On the other hand, we see that the expression of $d p$ with respect to (1.7) is

$$
\begin{aligned}
d p= & p_{\alpha \beta} \theta^{\beta} \wedge \theta^{\alpha}+\left(-p_{\alpha \bar{\beta}}+p_{\bar{\beta} \alpha}+i g_{\alpha \bar{\beta}} p_{0}\right) \theta^{\alpha} \wedge \theta^{\bar{\beta}}+p_{\bar{\alpha} \bar{\beta}} \theta^{\bar{\beta}} \wedge \theta^{\bar{\alpha}} \\
& +\left(-p_{\alpha 0}+p_{0 \alpha}-A_{\alpha \bar{\beta}} p_{\bar{\beta}}\right) \theta^{\alpha} \wedge \theta+\left(-p_{\bar{\alpha} 0}+p_{0 \bar{\alpha}}-A^{\beta} p_{\bar{\alpha}}\right) \theta^{\bar{\alpha}} \wedge \theta
\end{aligned}
$$

in virtue of (1.8) and (1.10). In particular, since $d^{2} \mu=0$, we obtain

$$
\mu_{\alpha \beta}=\mu_{\beta \alpha}, \quad \mu_{\alpha \bar{\beta}}-\mu_{\bar{\beta} \alpha}=i g_{\alpha \bar{\beta}} \mu_{0}, \quad \mu_{0 \alpha}-\mu_{\alpha 0}=A_{\alpha}^{\bar{\beta}} \mu_{\bar{\beta}} .
$$

Therefore we have the following lemma, by using Lemma 1.3:

Lemma 1.4. On $U \cap U^{\prime}$, the connection forms of $\nabla$ and $\nabla^{\prime}$ with respect to the admissible frame (1.6) are related as follows:

$$
\begin{aligned}
{ }^{\prime} v_{\beta}^{\gamma}= & v_{\beta}^{\gamma}+2\left(\mu_{\beta} \theta^{\gamma}-\mu^{\gamma} \theta_{\beta}\right)+2 \delta_{\beta}^{\gamma} \mu_{\alpha} \theta^{\alpha} \\
& +i\left(\mu_{\beta}^{\gamma}+\mu_{\beta}^{\gamma}+4 \mu_{\beta} \mu^{\gamma}+4 \delta_{\beta}^{\gamma} \mu_{\alpha} \mu^{\alpha}-i \delta_{\beta}^{\gamma} \mu_{0}\right) \theta .
\end{aligned}
$$

Remark. The equation (5.7) in [6] is the relation of components of the connection forms when $(1.17)$ is an adapted frame to $\left(U^{\prime},\left(\phi^{\prime}, \xi^{\prime}, \theta^{\prime}\right)\right)$ such that $Z_{\alpha}^{\prime}=\varepsilon e^{-\mu} Z_{\alpha}$ on $U \cap U^{\prime}$.

Let $R^{\nabla}$ be the curvature tensor of the Tanaka-Webster connection $\nabla$ associated with $(U,(\phi, \xi, \theta))$. Define $k^{\nabla}$ by

$$
k^{\nabla}(V, W)=\frac{1}{2} \operatorname{trace}\left(\phi R^{\nabla}(V, \phi W)\right)
$$

for all $V, W \in T M$ (cf. [10]), which is called the $*$-Ricci tensor of $\nabla$. Expressing $k^{\nabla}$ with respect to (1.6) and (1.7), we have

$$
\begin{aligned}
& k^{\nabla}(V, W)= \frac{1}{2}\left\{\theta\left(\phi R^{\nabla}(V, \phi W) \xi\right)+\theta^{\beta}\left(\phi R^{\nabla}(V, \phi W) Z_{\beta}\right)\right. \\
&\left.+\theta^{\bar{\beta}}\left(\phi R^{\nabla}(V, \phi W) Z_{\bar{\beta}}\right)\right\} \\
&= \frac{1}{2}\left\{i \theta^{\beta}\left(2\left(d v_{\beta}^{\gamma}+v_{\lambda}^{\gamma} \wedge v_{\beta}{ }^{\lambda}\right)(V, \phi W) Z_{\gamma}\right)\right. \\
& \quad-i \theta^{\bar{\beta}}\left(2\left(d v_{\bar{\beta}}^{\bar{\gamma}}+v_{\bar{\lambda}}^{\bar{\gamma}} \wedge v_{\bar{\beta}}^{\bar{\lambda}}\right)(V, \phi W) Z_{\bar{\gamma})\}}\right) \\
&=i\left(d v_{\alpha}{ }^{\alpha}-d v_{\bar{\alpha}}^{\bar{\alpha}}\right)(V, \phi W),
\end{aligned}
$$


where we have used the definition of $R^{\nabla}$ in [5] and the fact that $\phi R^{\nabla}=R^{\nabla} \phi$ proved in [10]. Note that $d v_{\alpha}{ }^{\alpha}$ is independent of the choice of frames adapted to $(U,(\phi, \xi, \theta))$. From (1.11), we have

$$
v_{\alpha}^{\alpha}+v_{\bar{\alpha}}^{\bar{\alpha}}=g^{\bar{\beta} \alpha} d g_{\alpha \bar{\beta}}=d \log \left|\operatorname{det}\left(g_{\alpha \bar{\beta}}\right)\right| .
$$

Therefore we obtain

$$
d v_{\alpha}^{\alpha}+d v_{\bar{\alpha}}^{\bar{\alpha}}=0
$$

which states that the complex valued 2-form $d v_{\alpha}{ }^{\alpha}$ is pure imaginary. By using this result, we see that the equation (1.26) becomes

$$
k^{\nabla}(V, W)=2 i d v_{\alpha}^{\alpha}(V, \phi W) .
$$

Remark. The $*$-Ricci tensor $k^{\nabla}$ satisfies the following equations (cf. [10]):

$$
k^{\nabla}(Y, Z)=k^{\nabla}(Z, Y), \quad k^{\nabla}(J Y, J Z)=k^{\nabla}(Y, Z) .
$$

for all $Y, Z \in \mathscr{D}$.

A $\mathscr{D}$-preserving almost contact structure $(U,(\phi, \xi, \theta))$ is pseudo-Einstein if $k^{\nabla}$ is proportional to $g$ pointwise, that is, there is a smooth function $\lambda$ on $U$ such that

$$
k^{\nabla}(Y, Z)=\lambda g(Y, Z)
$$

for all $Y, Z \in \mathscr{D}$.

For any two $\mathscr{D}$-preserving almost contact structures $(U,(\phi, \xi, \theta))$ and $\left(U^{\prime},\left(\phi^{\prime}, \xi^{\prime}, \theta^{\prime}\right)\right)$, we obtain

$$
\begin{aligned}
k^{\nabla^{\prime}}(Y, Z)= & k^{\nabla}(Y, Z)-2(n+2) d q^{*}(Y, J Z) \\
& -g(Y, Z)\left\{2(n+1) p^{*}\left(P^{*}\right)+\operatorname{trace}_{\mathscr{D}} \nabla P^{*}\right\}
\end{aligned}
$$

for all $Y, Z \in \mathscr{D}$ on $U \cap U^{\prime}$, where trace $\mathscr{D}$ denotes the trace on only $\mathscr{D}$. In fact, the equation (1.29) can be shown as follows. By a direct calculation, we have (1.30) $\left(\nabla_{Y} p^{*}\right)(Z)-\left(\nabla_{J Y} q^{*}\right)(Z)+p^{*}(\xi) g(J Y, Z)-2 d p^{*}(J Y, J Z)=2 d q^{*}(Y, J Z)$ for all $Y, Z \in \mathscr{D}$. Using Lemma 1.3, we have

$$
\begin{aligned}
k^{\nabla^{\prime}}(Y, Z)= & k^{\nabla}(Y, Z)-(n+2)\left\{\left(\nabla_{Y} p^{*}\right)(Z)-\left(\nabla_{J Y} q^{*}\right)(Z)+p^{*}(\xi) g(J Y, Z)\right\} \\
& -g(Y, Z)\left\{2(n+1) p^{*}\left(P^{*}\right)+\operatorname{trace}_{\mathscr{D}} \nabla P^{*}\right\},
\end{aligned}
$$

for the detail, see [10]. Applying (1.30) to the above equation, we have (1.29).

Remark. We extend $p^{*}$ and $q^{*}$ to complex linear forms and denote them also by $p^{*}$ and $q^{*}$ respectively. Then the complex valued 1 -form $p^{*}+i q^{*}$ becomes a $(1,0)$-form, that is, $\left(p^{*}+i q^{*}\right)\left(\bar{Y}_{1}\right)=0$ for every $Y_{1} \in \mathscr{D}^{1,0}$. From the condition (C), we have $d\left(p^{*}+i q^{*}\right)\left(\bar{Y}_{1}, \bar{Y}_{2}\right)=0$ for all $Y_{1}, Y_{2} \in \mathscr{D}^{1,0}$. This fact is equivalent to the following equation: 


$$
d p^{*}(X, Y)-d p^{*}(J X, J Y)-d q^{*}(X, J Y)-d q^{*}(J X, Y)=0
$$

for all $X, Y \in \mathscr{D}$. Since $d p^{*}=d^{2} \mu=0$, the equation (1.31) states that $d q^{*}(J Y, J Z)=d q^{*}(Y, Z)$ for all $Y, Z \in \mathscr{D}$.

Let $s^{\nabla}$ be the usual Ricci tensor of the Tanaka-Webster connection $\nabla$ associated with $(U,(\phi, \xi, \theta))$, that is,

$$
s^{\nabla}(V, W)=\operatorname{trace}\left(X \rightarrow R^{\nabla}(X, V) W\right)
$$

for $V, W \in T M$. Define $S^{\nabla} \in \Gamma\left(U, \mathscr{D} \otimes \mathscr{D}^{*}\right)$ by $g\left(S^{\nabla} Y, Z\right)=s^{\nabla}(Y, Z)$ for all $Y, Z \in \Gamma(U, \mathscr{D})$. Moreover, define $\rho^{\nabla}$ by $\rho^{\nabla}=\operatorname{trace}_{\mathscr{D}} S^{\nabla}$, which is a smooth function on $U$ and is called the scalar curvature of $\nabla$ (cf. [10]). follows:

Remark. The Webster scalar curvature $R$ defined in [6] is related to $\rho^{\nabla}$ as

$$
\rho^{\nabla}=2 R
$$

On $U \cap U^{\prime}$, the scalar curvature $\rho^{\nabla^{\prime}}$ of $\nabla^{\prime}$ is related to $\rho^{\nabla}$ of $\nabla$ as follows:

$$
\varepsilon e^{2 \mu} \rho^{\nabla^{\prime}}=\rho^{\nabla}-4(n+1) \operatorname{trace}_{\mathscr{D}} \nabla P^{*}-4 n(n+1) p^{*}\left(P^{*}\right)
$$

(cf. [10]). By using the admissible frame (1.6), we have

$$
\operatorname{trace}_{\mathscr{D}} \nabla P^{*}=\mu_{\alpha}^{\alpha}+\mu_{\alpha}^{\alpha}=\mu_{\alpha}^{\alpha}+\mu_{\bar{\alpha}}{ }^{\bar{\alpha}}, \quad p^{*}\left(P^{*}\right)=2 \mu_{\alpha} \mu^{\alpha},
$$

so that we obtain

$$
\varepsilon e^{2 \mu} \rho^{\nabla^{\prime}}=\rho^{\nabla}-4(n+1)\left\{\mu_{\alpha}^{\alpha}+\mu_{\alpha}^{\alpha}+2 n \mu_{\alpha} \mu^{\alpha}\right\} .
$$

Note that $\mu_{\alpha}^{\alpha}, \mu_{\alpha}{ }^{\alpha}$ and $\mu_{\alpha} \mu^{\alpha}$ are independent of the choice of the admissible frames of $(U,(\phi, \xi, \theta))$.

\section{CR Weyl connections}

Let $(M, \mathscr{D}, J)$ be a nondegenerate CR manifold of dimension $2 n+1(n \geq 1)$. A linear connection $D$ on $M$ is a $C R$ Weyl connection if for each $\mathscr{D}$-preserving almost contact structure $(U,(\phi, \xi, \theta))$, there is a 1 -form $p$ on $U$ such that

$$
\begin{gathered}
D_{V} \theta=-2 p(V) \theta, \quad D_{V \varsigma}=2 p(V) \varsigma, \quad D_{V}^{\circ} J=0, \quad D_{V}^{\circ} g=-2 p(V) g, \\
T(X, Y)=-\omega(X, Y) \varsigma, \quad T(\varsigma, Y)=-\frac{1}{2} \varphi\left(\mathscr{L}_{\varsigma} \varphi\right) Y
\end{gathered}
$$

for all $V \in T M$ and $X, Y \in \mathscr{D}$, where $D^{\circ}$ denotes the induced connection on the hyperdistribution $\mathscr{D}, T$ the torsion tensor of $D, \varsigma=\xi-2 Q, \varphi=\phi-2 \theta \otimes P$, $P \in \Gamma(U, \mathscr{D})$ is defined by $g(P, X)=p(X)$ for every $X \in \Gamma(U, \mathscr{D})$ and $Q=J P$. For a given $D$, we call $p$ the associated 1 -form relative to $(U,(\phi, \xi, \theta))$. Let a CR Weyl connection $D$ on $M$ be fixed. Then it is shown that each associated 1-form $p$ should satisfy 


$$
d p(J X, J Y)+d p(X, Y)=0
$$

for all $X, Y \in \mathscr{D}$ (cf. [8]). Taking any two $\mathscr{D}$-preserving almost contact structures $(U,(\phi, \xi, \theta))$ and $\left(U^{\prime},\left(\phi^{\prime}, \xi^{\prime}, \theta^{\prime}\right)\right)$,

$$
\begin{aligned}
-2 p^{\prime}(V) \theta^{\prime}=D_{V} \theta^{\prime}=D_{V}\left(\varepsilon e^{2 \mu} \theta\right) & =2 \varepsilon e^{2 \mu} d \mu(V) \theta+\varepsilon e^{2 \mu} D_{V} \theta \\
& =2 d \mu(V) \theta^{\prime}+\varepsilon e^{2 \mu}(-2 p(V) \theta) \\
& =-2(p-d \mu)(V) \theta^{\prime}
\end{aligned}
$$

so that we have

$$
p^{\prime}=p-d \mu
$$

on $U \cap U^{\prime}$, where $p^{\prime}$ is the associated 1 -form on $U^{\prime}$ relative to $\left(U^{\prime},\left(\phi^{\prime}, \xi^{\prime}, \theta^{\prime}\right)\right)$. From (2.3), we have

$$
P^{\prime}=\varepsilon e^{-2 \mu}\left(P-P^{*}\right), \quad Q^{\prime}=\varepsilon e^{-2 \mu}\left(Q-Q^{*}\right)
$$

on $U \cap U^{\prime}$. Therefore we have

$$
\xi^{\prime}-2 Q^{\prime}=\varepsilon e^{-2 \mu}(\xi-2 Q), \quad \phi^{\prime}-2 \theta^{\prime} \otimes P^{\prime}=\phi-2 \theta \otimes P
$$

because of (1.12), so that we obtain

$$
\varsigma^{\prime}=\varepsilon e^{-2 \mu} \varsigma, \quad \varphi^{\prime}=\varphi
$$

on $U \cap U^{\prime}$.

Let $\nabla$ be the Tanaka-Webster connection associated with $(U,(\phi, \xi, \theta))$. Then the following lemma has been proved in [8].

Lemma 2.1. Define the difference $H$ between $D$ and $\nabla$ by $H=D-\nabla$. Then, on $U$, we have

$$
\begin{gathered}
H(X, Y)=p(X) Y+p(Y) X-g(X, Y) P \\
+q(X) J Y+q(Y) J X-g(J X, Y) Q, \\
H(\xi, Y)=\nabla_{J Y} P+\nabla_{Y} Q-2 q(Y) P+2 p(Y) Q+2 g(P, P) J Y
\end{gathered}
$$

for all $X, Y \in \Gamma(U, \mathscr{D})$, where $p$ is the associated 1 -form relative to $(U,(\phi, \xi, \theta))$ and $q=-p \circ \phi$.

Next lemma gives us the sufficient condition for the existence of a CR Weyl connection.

Lemma 2.2. Assume that $M$ be covered by open sets $\{U\}$ and that, for each $\mathscr{D}$-preserving almost contact structure $(U,(\phi, \xi, \theta))$, there is a 1-form $p$ on each $U$ satisfying the equation (2.2) and the 1-forms satisfy (2.3) on each nonempty intersection $U \cap U^{\prime}$. Then there is a CR Weyl connection $D$ on $M$ such that the associated 1-form coincides with the given $p$ on each $U$. 
Proof. For each $(U,(\phi, \xi, \theta))$, define $D$ by

$$
D_{X} Y=\nabla_{X} Y+H(X, Y), \quad D_{\xi} Y=\nabla_{\xi} Y+H(\xi, Y), \quad D_{V} \varsigma=2 p(V) \varsigma
$$

for all $V \in T U$ and $X, Y \in \Gamma(\mathscr{D})$, where $H$ is defined as (2.6) and (2.7). Then $D$ becomes a linear connection on the open submanifold $U$. By a direct calculation, we can show that the linear connection $D$ satisfies (2.1). Note that we require the assumption (2.2) to show $D_{\xi}^{\circ} g=-2 p(\xi) g$.

We can complete the proof if, for any two $\mathscr{D}$-preserving almost contact structures $(U,(\phi, \xi, \theta))$ and $\left(U^{\prime},\left(\phi^{\prime}, \xi^{\prime}, \theta^{\prime}\right)\right)$, we show

$$
D^{\prime}=D \text {. }
$$

on $U \cap U^{\prime}$. From (1.12), (2.3) and the definition of $q$, we have

$$
q^{\prime}=q-q^{*}+2 p\left(P^{*}\right) \theta-2 p^{*}\left(P^{*}\right) \theta .
$$

First, applying (1.13), (2.3), (2.4) and (2.10) to (2.8), we have

$$
D_{X}^{\prime} Y=D_{X} Y
$$

for all $X, Y \in \Gamma\left(U \cap U^{\prime}, \mathscr{D}\right)$. Next, from (1.13), (1.14) and (2.4), we have the two following equations:

$$
\begin{aligned}
\nabla_{J X}^{\prime} P^{\prime}=\varepsilon e^{-2 \mu} & \left\{\nabla_{J X} P-\nabla_{J X} P^{*}-2 q^{*}(X) P^{*}-2 p^{*}(X) Q^{*}\right. \\
& +q^{*}(X) P+q(X) P^{*}+p^{*}(X) Q+p(X) Q^{*} \\
& \left.+p^{*}(P) J X-q^{*}(P) X-p^{*}\left(P^{*}\right) J X\right\} \\
\nabla_{X}^{\prime} Q^{\prime}=\varepsilon e^{-2 \mu} & \left\{\nabla_{X} Q-\nabla_{X} Q^{*}+2 q^{*}(X) P^{*}+2 p^{*}(X) Q^{*}\right. \\
& -q^{*}(X) P-q(X) P^{*}-p^{*}(X) Q-p(X) Q^{*} \\
& \left.+p^{*}(P) J X+p^{*}(Q) X-p^{*}\left(P^{*}\right) J X\right\}
\end{aligned}
$$

for every $X \in \Gamma\left(U \cap U^{\prime}, \mathscr{D}\right)$. Applying these equations to (2.8), we obtain

$$
D_{\xi^{\prime}}^{\prime} X=D_{\xi^{\prime}} X \text {. }
$$

Finally, we have

$$
\begin{aligned}
D_{V}^{\prime} \xi^{\prime} & =D_{V}^{\prime}\left(\varsigma^{\prime}+2 Q^{\prime}\right) \\
& =2 p^{\prime}(V) \varsigma^{\prime}-D_{V}\left(\xi^{\prime}-2 Q^{\prime}\right)+D_{V} \xi^{\prime} \\
& =2 p^{\prime}(V) \varsigma^{\prime}-D_{V}\left(\varepsilon e^{-2 \mu} \varsigma\right)+D_{V} \xi^{\prime} \\
& =D_{V} \xi^{\prime},
\end{aligned}
$$

where we have used (2.5), (2.11) and (2.14).

From the definition of CR Weyl connections and Lemma 2.2, we see that there is a CR Weyl connection $D$ if and only if, for a family of $\mathscr{D}$-preserving almost contact structures $(U,(\phi, \xi, \theta))$ such that $\{U\}$ cover $M$, there is a 1 -form 
$p$ on each $U$ which satisfies (2.2) and (2.3) on every nonempty intersection $U \cap U^{\prime}$.

If $M$ is orientable, there is a global $\mathscr{D}$-preserving almost contact structure $(\phi, \xi, \theta)$. Then we have the following corollary (cf. [8]):

COROLlary 2.3. Let $M$ be an orientable nondegenerate $C R$ manifold. Then, for a 1-form $p$ on $M$ satisfying (2.2), there is a CR Weyl connection $D$ whose associated form is $p$.

Proof. Fix a global $\mathscr{D}$-preserving almost contact structure $(\phi, \xi, \theta)$ and define $D$ by (2.8). Then the linear connection $D$ on $M$ is a CR Weyl connection.

Note that if we define $D^{\prime}$ by using the global 1 -form $p-d \mu$ and a $\mathscr{D}$ preserving almost contact structure $\left(\phi^{\prime}, \xi^{\prime}, \theta^{\prime}\right)$ related with $(\phi, \xi, \theta)$ as $(1.12)$, we see that $D^{\prime}$ coincides with $D$. However if we use the 1 -form $p$ and $\left(\phi^{\prime}, \xi^{\prime}, \theta^{\prime}\right)$, we obtain another CR Weyl connection.

Remark. Let $D$ be a CR Weyl connection on $M$. If each local 1-form associated with $D$ is exact, there is a $\mathscr{D}$-preserving almost contact structure $(U,(\phi, \xi, \theta))$ such that $\nabla=D$ on $U$, where $\nabla$ is the Tanaka-Webster connection determined by $(U,(\phi, \xi, \theta))$.

Let $D$ be a CR Weyl connection on $M$ and $R$ the curvature tensor of $D$. Define $k$ by

$$
k(V, W)=\frac{1}{2} \operatorname{trace}(\varphi R(V, \varphi W))
$$

for all $V, W \in T M$, which is called the $*$-Ricci tensor of $D$. Since $\varphi^{\prime}=\varphi$ from (2.5), $k$ is globally defined. It was shown in [8] that the tensor field $k$ satisfies

$$
\begin{aligned}
& k(X, Y)-k(Y, X)=-4(n+2) d p(X, Y), \\
& k(J X, J Y)-k(X, Y)=4(n+2) d p(X, Y)
\end{aligned}
$$

for all $X, Y \in \mathscr{D}$. Note that the 2-form $d p$ globally exists on $M$ because of (2.3). We denote the usual Ricci tensor of $D$ by $s$ and define $S \in \Gamma\left(U, \mathscr{D} \otimes \mathscr{D}^{*}\right)$ by $g(S Y, Z)=s(Y, Z)$ for all $Y, Z \in \Gamma(U, \mathscr{D})$ for each $\mathscr{D}$-preserving almost contact structure $(U,(\phi, \xi, \theta))$. Moreover define $\rho$ by $\rho=\operatorname{trace}_{\mathscr{D}} S$. Then, for any two $\mathscr{D}$-preserving almost contact structures $(U,(\phi, \xi, \theta))$ and $\left(U^{\prime},\left(\phi^{\prime}, \xi^{\prime}, \theta^{\prime}\right)\right)$, we have

$$
\rho^{\prime}=\varepsilon e^{-2 \mu} \rho
$$

on $U \cap U^{\prime}$. Also, for $(U,(\phi, \xi, \theta))$, define $l$ by

$$
l(Y, Z)=-\frac{1}{2(n+2)} k(Y, Z)+\frac{1}{8(n+1)(n+2)} \rho g(Y, Z)
$$


for any $Y, Z \in \mathscr{D}$. We see from (1.12) and (2.17) that $l$ is globally defined on $M$. We have

$$
\begin{gathered}
l(Y, Z)-l(Z, Y)=2 d p(Y, Z), \\
l(J Y, J Z)-l(Y, Z)=-2 d p(Y, Z)
\end{gathered}
$$

for all $Y, Z \in \mathscr{D}$ because of (2.16). Define ric $\in \Gamma\left(\mathscr{D}^{*} \otimes \mathscr{D}^{*}\right)$ by

$$
\operatorname{ric}(Y, Z)=l(Y, Z)-d p(Y, Z)
$$

for $Y, Z \in \mathscr{D}$. From (2.19) we have

$$
\operatorname{ric}(Y, Z)=\operatorname{ric}(Z, Y), \quad \operatorname{ric}(J Y, J Z)=\operatorname{ric}(Y, Z)
$$

for all $Y, Z \in \mathscr{D}$. A CR Weyl connection $D$ is $C R$ Einstein-Weyl if, for each $\mathscr{D}$ preserving almost contact structure $(U,(\phi, \xi, \theta))$, there is a smooth function $\lambda$ on $U$ such that

$$
\operatorname{ric}(Y, Z)=\lambda g(Y, Z)
$$

for all $Y, Z \in \mathscr{D}$. Since ric is globally defined, for any two $\mathscr{D}$-preserving almost contact structures $(U,(\phi, \xi, \theta))$ and $\left(U^{\prime},\left(\phi^{\prime}, \xi^{\prime}, \theta^{\prime}\right)\right)$, we have

$$
\lambda^{\prime}=\varepsilon e^{-2 \mu} \lambda \text { on } U \cap U^{\prime}
$$

Let $D$ be a CR Weyl connection on $M$. For every $\mathscr{D}$-preserving almost contact structure $(U,(\phi, \xi, \theta))$, we have

$$
\begin{aligned}
k(Y, Z)= & k^{\nabla}(Y, Z)-2(n+2) d q(Y, J Z) \\
& -g(Y, Z)\left\{2(n+1) p(P)+\operatorname{trace}_{\mathscr{D}} \nabla P\right\}
\end{aligned}
$$

for all $Y, Z \in \mathscr{D}$ on $U$. In fact, this equation is showed by the same way as (1.29). In [8] it was shown that

$$
\begin{aligned}
k(Y, Z)= & k^{\nabla}(Y, Z)-g(Y, Z)\left\{2(n+1) p(P)+\operatorname{trace}_{\mathscr{D}} \nabla P\right\} \\
& -(n+2)\left\{\left(\nabla_{Y} p\right)(Z)-\left(\nabla_{J Y} q\right)(Z)+p(\xi) g(J Y, Z)+2 d p(Y, Z)\right\}
\end{aligned}
$$

for all $Y, Z \in \mathscr{D}$ on $U$. Also by a direct calculation, we have the equation in which we replace $p^{*}$ and $q^{*}$ with $p$ and $q$ in the equation (1.30) respectively. Applying (2.2) and this equation to (2.25), we obtain (2.24).

Remark. Since the complex valued 1 -form $p+i q$ is a $(1,0)$-form, we have the equation in which we replace $p^{*}$ and $q^{*}$ with $p$ and $q$ in the equation (1.31) respectively. Applying (2.2) to this equation, we have

$$
2 d p(Y, Z)=d q(Y, J Z)+d q(J Y, Z)
$$

for all $X, Y \in \mathscr{D}$.

It is also proved in [8] that 


$$
\rho=\rho^{\nabla}-4(n+1) \operatorname{trace}_{\mathscr{D}} \nabla P-4 n(n+1) p(P)
$$

on $U$.

For a $\mathscr{D}$-preserving almost contact structure $(U,(\phi, \xi, \theta))$, define $l^{\nabla}$ by

$$
l^{\nabla}(Y, Z)=-\frac{1}{2(n+2)} k^{\nabla}(Y, Z)+\frac{1}{8(n+1)(n+2)} \rho^{\nabla} g(Y, Z)
$$

for all $Y, Z \in \mathscr{D}$ on $U$. Then, from (2.24) and (2.27), we have

$$
l(Y, Z)=l^{\nabla}(Y, Z)+d q(Y, J Z)+\frac{1}{2} p(P) g(Y, Z)
$$

on $U$. Then, from $(2.20),(2.26)$ and (2.28), we have

$$
\begin{aligned}
\operatorname{ric}(Y, Z)= & -\frac{1}{2(n+2)} k^{\nabla}(Y, Z)+\frac{1}{2}\{d q(Y, J Z)-d q(J Y, Z)\} \\
& +\left\{\frac{1}{2} p(P)+\frac{1}{8(n+1)(n+2)} \rho^{\nabla}\right\} g(Y, Z)
\end{aligned}
$$

on $U$. If a CR Weyl connection $D$ is CR Einstein-Weyl, for every $\mathscr{D}$-preserving almost contact structure $(U,(\phi, \xi, \theta))$, we obtain

$$
\begin{aligned}
& k^{\nabla}(Y, Z)-(n+2)\{d q(Y, J Z)-d q(J Y, Z)\} \\
& \quad=-2(n+2)\left\{\lambda-\frac{1}{2} p(P)-\frac{1}{8(n+1)(n+2)} \rho^{\nabla}\right\} g(Y, Z)
\end{aligned}
$$

for all $Y, Z \in \mathscr{D}$ in virtue of (2.29). Conversely, from Lemma 2.2 and (2.29), we immediately have

Lemma 2.4. Let $p$ be the local 1-form on each $U$ in the assumption of Lemma 2.2. If, for each $\mathscr{D}$-preserving almost contact structure $(U,(\phi, \xi, \theta))$, there is a smooth function $\Lambda$ on $U$ such that

$$
k^{\nabla}(Y, Z)-(n+2)\{d q(Y, J Z)-d q(J Y, Z)\}=\Lambda g(Y, Z)
$$

for all $Y, Z \in \mathscr{D}$, then the $C R$ Weyl connection D determined by Lemma 2.2 is $C R$ Einstein-Weyl.

If $M$ is orientable, we have (cf. [8])

Corollary 2.5. Assume that there is a 1-form $p$ on $M$ satisfying (2.2). If there is a $\mathscr{D}$-preserving almost contact structure $(\phi, \xi, \theta)$ such that $(2.31)$ holds, a CR Weyl connection D in Corollary 2.3 is CR Einstein-Weyl.

\section{CR Einstein-Weyl structures on an orientable CR manifold}

In this section, let $M$ be an orientable nondegenerate $\mathrm{CR}$ manifold of dimension $2 n+1(n \geq 1)$. Then there exist global $\mathscr{D}$-preserving almost contact 
structures. We choose $(\phi, \xi, \theta)$ from them arbitrarily. The Tanaka-Webster connection $\nabla$ associated with $(\phi, \xi, \theta)$ is a linear connection defined on $M$.

Let $K_{M}$ be the complex line bundle consisting of $(n+1,0)$-forms which vanish on $\mathscr{D}^{0,1}$, that is,

$$
\left.K_{M}=\left\{\zeta \in \bigwedge^{n+1} \mathbf{C} T^{*} M \mid \bar{Y}\right\rfloor \zeta=0 \text { for } Y \in \mathscr{D}^{1,0}\right\}
$$

which is called the canonical bundle on $M$. Farris [2] showed the following lemma which we prove to complete this paper.

Lemma 3.1. For every nonvanishing global cross section $\zeta$ of $K_{M}$, there exists a unique 1-form $\theta^{\prime}$ annihilating $\mathscr{D}$ up to the multiple of \pm 1 such that

$$
\left.\left.\theta^{\prime} \wedge\left(d \theta^{\prime}\right)^{n}=i^{n^{2}} n !(-1)^{t^{\prime}} \theta^{\prime} \wedge\left(\xi^{\prime}\right\rfloor \zeta\right) \wedge\left(\xi^{\prime}\right\rfloor \bar{\zeta}\right)
$$

where $\xi^{\prime}$ is a unique vector field satisfying the condition $(*)$ determined by $\theta^{\prime}$ and $t^{\prime}$ denotes the signature of the Levi form defined by $\theta^{\prime}$.

Proof. We first show the existence of the 1-form $\theta^{\prime}$. We fix a 1 -form $\theta$ on $M$ annihilating $\mathscr{D}$ and put $\theta^{\prime}=\varepsilon e^{2 \mu} \theta$. We shall find a function $\mu$ such that the 1 -form $\theta^{\prime}$ satisfies the equation (3.2).

Assume that the 1 -form $\theta^{\prime}$ satisfies (3.2). Take an admissible frame (1.6) and the dual frame $(1.7)$ of $(\phi, \xi, \theta)$ on an open set $U \subset M$ and determine the admissible frame (1.17) and the dual frame (1.18) of $\left(\phi^{\prime}, \xi^{\prime}, \theta^{\prime}\right)$ on the same $U$. From (1.8), we have

$$
(d \theta)^{n}=i^{n^{2}} n ! \operatorname{det}\left(g_{\alpha \bar{\beta}}\right) \theta^{1} \wedge \cdots \wedge \theta^{n} \wedge \theta^{\overline{1}} \wedge \cdots \wedge \theta^{\bar{n}}
$$

on $U$. It follows that the equation (3.3) similarly holds for every 1-form $\theta$ annihilating $\mathscr{D}$ and every admissible coframe of $(\phi, \xi, \theta)$. Also when we put the local expressions of $\zeta$ with respect to (1.7) and (1.18) as

$$
\zeta=f \theta \wedge \theta^{1} \wedge \cdots \wedge \theta^{n}, \quad \zeta=f^{\prime} \theta^{\prime} \wedge^{\prime} \theta^{1} \wedge \cdots \wedge^{\prime} \theta^{n}
$$

on $U$ respectively, we have from (1.19)

$$
f^{\prime}=\varepsilon e^{-2 \mu} f
$$

Since the 1-form $\theta^{\prime}$ satisfies (3.2), we have

$$
(-1)^{t^{\prime}} \operatorname{det}\left(g_{\alpha \bar{\beta}}^{\prime}\right)=\left|f^{\prime}\right|^{2}
$$

on $U$, where we have used (3.3) and (3.4). Applying (3.5) and the equation $g_{\alpha \bar{\beta}}^{\prime}=\varepsilon e^{2 \mu} g_{\alpha \bar{\beta}}$ to (3.6), we have

$$
(-1)^{t+t^{\prime}}\left(\varepsilon e^{2 \mu}\right)^{n+2}=\frac{|f|^{2}}{(-1)^{t} \operatorname{det}\left(g_{\alpha \bar{\beta}}\right)},
$$

where $t$ denotes the signature of the Levi form defined by $\theta$. Note that if $\varepsilon=1$ (resp. $\varepsilon=-1), t^{\prime}=t\left(\right.$ resp. $\left.t^{\prime}=n-t\right)$. Thus the left hand side of (3.7) becomes $\left(e^{2 \mu}\right)^{n+2}$. Therefore we obtain 


$$
\mu=\frac{1}{2(n+2)} \log \left(\frac{|f|^{2}}{(-1)^{t} \operatorname{det}\left(g_{\alpha \bar{\beta}}\right)}\right)
$$

on $U$.

Conversely, define a function $\mu$ by (3.8) on each open set $U$ where an admissible frame $(1.6)$ of $(\phi, \xi, \theta)$ is defined. Taking another admissible frame and the dual frame

$$
\left\{\xi, \tilde{Z}_{1}, \ldots, \tilde{Z}_{n}, \tilde{Z}_{\overline{1}}, \ldots, \tilde{Z}_{\bar{n}}\right\}, \quad\left\{\theta, \tilde{\theta}^{1}, \ldots, \tilde{\theta}^{n}, \tilde{\theta}^{\overline{1}}, \ldots, \tilde{\theta}^{\bar{n}}\right\}
$$

on $\tilde{U}$, we have $\tilde{Z}_{\alpha}=Z_{\beta} X_{\alpha}{ }^{\beta}$ on $U \cap \tilde{U}$, where $\left(X_{\alpha}{ }^{\beta}\right)$ is the $n \times n$ nonsingular matrix valued function on $U \cap \tilde{U}$ and it is written as $X=\left(X_{\alpha}^{\beta}\right)$. Then we obtain $\tilde{f}=f \operatorname{det} X$ and $\left(\tilde{g}_{\alpha \bar{\beta}}\right)={ }^{t} X\left(g_{\alpha \bar{\beta}}\right) \bar{X}$ on $U \cap \tilde{U}$. Thus we have $\tilde{\mu}=\mu$ on $U \cap \tilde{U}$, which states that the function $\mu$ is a globally defined function on $M$. Putting $\theta^{\prime}=\varepsilon e^{2 \mu} \theta$, we see that the 1 -form $\theta^{\prime}$ satisfies (3.2).

Next, we show the uniqueness of the 1-form $\theta^{\prime}$. Assume that two 1-forms $\theta$ and $\theta^{\prime}$ annihilating $\mathscr{D}$ satisfy (3.2). Then there is a smooth function $\mu$ such that $\theta^{\prime}=\varepsilon e^{2 \mu} \theta$ by Lemma 1.2. Since both $\theta^{\prime}$ and $\theta$ satisfy (3.2), we obtain (3.7) and the equation $(-1)^{t} \operatorname{det}\left(g_{\alpha \bar{\beta}}\right)=|f|^{2}$ on an arbitrary open set $U$ where an admissible frame (1.6) of $(\phi, \xi, \theta)$ and (1.17) of $\left(\phi^{\prime}, \xi^{\prime}, \theta^{\prime}\right)$ are defined. Therefore we have $e^{2 \mu}=1$.

Lee [7] showed the following lemma. We show this Lee's result by the other way, since the proof makes us guess the main theorem in this section.

Lemma 3.2. Assume that there exists a closed nonvanishing global cross section $\zeta$ of $K_{M}$. Let $\theta^{\prime}$ be a 1-form on $M$ determined by $\zeta$ as in Lemma 3.1. Then the D-preserving almost contact structure $\left(\phi^{\prime}, \xi^{\prime}, \theta^{\prime}\right)$ determined by $\theta^{\prime}$ is pseudo-Einstein.

Proof. We fix a $\mathscr{D}$-preserving almost contact structure $(\phi, \xi, \theta)$ on $M$ and put $\theta^{\prime}=e^{2 \mu} \theta$ by using the smooth function $\mu$ defined by (3.8) for $(\phi, \xi, \theta)$. Take an arbitrary admissible frame (1.6) and the dual frame (1.7) on an open set $U$. To show that $\left(\phi^{\prime}, \xi^{\prime}, \theta^{\prime}\right)$ is pseudo-Einstein by making use of (1.28) and (1.29), it is sufficient to show that there is a function $\Lambda$ such that

$$
i d v_{\alpha}{ }^{\alpha}-(n+2) d q^{*} \equiv \Lambda d \theta \quad(\bmod \theta),
$$

where $v_{\beta}^{\gamma}$ is the connection form of the Tanaka-Webster connection associated with $(\phi, \xi, \theta)$ with respect to (1.6), $q^{*}=-p^{*} \circ \phi$ and $p^{*}=d \mu$. Note that since $k^{\nabla}(J Y, J Z)=k^{\nabla}(Y, Z)$ and $d q^{*}(J Y, J Z)=d q^{*}(Y, Z)$ for $Y, Z \in \mathscr{D}, d v_{\alpha}{ }^{\alpha}$ and $d q^{*}$ do not have the parts of $\theta^{\beta} \wedge \theta^{\gamma}$ and $\theta^{\bar{\beta}} \wedge \theta^{\bar{\gamma}}$.

From (1.11) and the fact that $d \operatorname{det}\left(g_{\alpha \bar{\beta}}\right)=\operatorname{det}\left(g_{\alpha \bar{\beta}}\right) g^{\bar{\lambda} \gamma} d g_{\gamma \bar{\lambda}}$, we have

$$
p^{*}=\frac{1}{n+2} \operatorname{Re}\left(\frac{d f}{f}-v_{\alpha}^{\alpha}\right),
$$


where $\mathrm{Re}$ denotes the real parts of complex valued forms. To simplify the notation, we put $v=v_{\alpha}{ }^{\alpha}$. In general, giving a 1 -form $p=\operatorname{Re}(\alpha)$ for a complex valued 1 -form $\alpha=\alpha_{\beta} \theta^{\beta}+\alpha_{\bar{\beta}} \theta^{\bar{\beta}}+\alpha_{0} \theta$ on $U$, we have

$$
q=\operatorname{Im}\left(\alpha_{\beta} \theta^{\beta}-\alpha_{\bar{\beta}} \theta^{\bar{\beta}}\right),
$$

where $q=-p \circ \phi$ and Im denotes the imaginary parts of complex valued forms. Thus from (3.11) and (3.12), we have

$$
q^{*}=\frac{1}{n+2} \operatorname{Im}\left\{\left(\frac{f_{\beta}}{f}-v_{\beta}\right) \theta^{\beta}-\left(\frac{f_{\bar{\beta}}}{f}-v_{\bar{\beta}}\right) \theta^{\bar{\beta}}\right\},
$$

where

$$
d f=f_{\beta} \theta^{\beta}+f_{\bar{\beta}} \theta^{\bar{\beta}}+f_{0} \theta, \quad v=v_{\beta} \theta^{\beta}+v_{\bar{\beta}} \theta^{\bar{\beta}}+v_{0} \theta .
$$

Note that although both $d f / f$ and $v$ depend on the choice of the admissible frames of $(\phi, \xi, \theta), d f / f-v$ and $d(d f / f)$ are independent of the choice of them. The equation $d d f=0$ yields

$$
\left(-f_{\beta \bar{\gamma}}+f_{\bar{\gamma} \beta}+i g_{\beta \bar{\gamma}} f_{0}\right) \theta^{\beta} \wedge \theta^{\bar{\gamma}} \equiv 0 .
$$

Since

$$
\begin{aligned}
d\left\{\left(\frac{f_{\bar{\beta}}}{f}-v_{\bar{\beta}}\right) \theta^{\bar{\beta}}\right\} & \left(\frac{1}{f} f_{\bar{\beta} \gamma}-\frac{1}{f^{2}} f_{\bar{\beta}} f_{\gamma}-v_{\bar{\beta} \gamma}\right) \theta^{\gamma} \wedge \theta^{\bar{\beta}}+\left(\frac{1}{f} f_{\bar{\beta} \bar{\gamma}}-\frac{1}{f^{2}} f_{\bar{\beta}} f_{\bar{\gamma}}-v_{\bar{\beta} \bar{\gamma}}\right) \theta^{\bar{\gamma}} \wedge \theta^{\bar{\beta}} \\
& -\left(\frac{1}{f} f_{\bar{\beta} 0}-\frac{1}{f^{2}} f_{\bar{\beta}} f_{0}-v_{\bar{\beta} 0}\right) \theta^{\bar{\beta}} \wedge \theta-\left(\frac{f_{\bar{\gamma}}}{f}-v_{\bar{\gamma}}\right) A^{\bar{\gamma}}{ }_{\beta} \theta^{\beta} \wedge \theta
\end{aligned}
$$

and $d\left\{\left(f_{\beta} / f-v_{\beta}\right) \theta^{\beta}\right\}$ is similarly calculated, we have

$$
\begin{aligned}
& d q^{*} \equiv \frac{1}{2 i(n+2)}\left\{-\frac{1}{f} f_{\beta \bar{\gamma}}-\frac{1}{f} f_{\bar{\gamma} \beta}+\frac{2}{f^{2}} f_{\beta} f_{\bar{\gamma}}+v_{\beta \bar{\gamma}}+v_{\bar{\gamma} \beta}\right. \\
& \left.-\frac{1}{\bar{f}} \overline{f_{\gamma \bar{\beta}}}-\frac{1}{\bar{f}} \overline{f_{\bar{\beta} \gamma}}+\frac{2}{\bar{f}^{2}} \overline{f_{\gamma}} \overline{f_{\bar{\beta}}}+\overline{v_{\gamma \bar{\beta}}}+\overline{v_{\bar{\beta} \gamma}}\right\} \theta^{\beta} \wedge \theta^{\bar{\gamma}} \quad(\bmod \theta) .
\end{aligned}
$$

On the other hand, from the assumption $d \zeta=0$, we have

$$
\left(\frac{1}{f} f_{\bar{\beta}}-v_{\bar{\beta}}\right) \theta^{\bar{\beta}} \equiv 0,
$$

where we have used (1.10). Therefore (3.15) vanishes identically. In particular we have

$$
\frac{1}{f} f_{\bar{\gamma} \beta}-\frac{1}{f^{2}} f_{\bar{\gamma}} f_{\beta}-v_{\bar{\gamma} \beta}=0
$$


Applying (3.14) and (3.17) to (3.16), we have

$$
d q^{*} \equiv \frac{1}{2 i(n+2)}\left\{v_{\beta \bar{\gamma}}-v_{\bar{\gamma} \beta}+\overline{v_{\gamma \bar{\beta}}}-\overline{v_{\bar{\beta} \gamma}}-2 i \operatorname{Im}\left(\frac{f_{0}}{f}\right) i g_{\beta \bar{\gamma}}\right\} \theta^{\beta} \wedge \theta^{\bar{\gamma}} \quad(\bmod \theta) .
$$

Also, from (1.22), we have

$$
\begin{aligned}
& \left(v_{\beta \bar{\gamma}}-v_{\bar{\gamma} \beta}\right) \theta^{\beta} \wedge \theta^{\bar{\gamma}} \equiv-d v+i g_{\beta \bar{\gamma}} v_{0} \theta^{\beta} \wedge \theta^{\bar{\gamma}}(\bmod \theta), \\
& \left(\overline{v_{\gamma \bar{\beta}}}-\overline{v_{\bar{\beta} \gamma}}\right) \theta^{\beta} \wedge \theta^{\bar{\gamma}} \equiv d \bar{v}-i g_{\beta \bar{\gamma}} \bar{\nu}_{0} \theta^{\beta} \wedge \theta^{\bar{\gamma}}(\bmod \theta) .
\end{aligned}
$$

Substituting (3.19) and applying the fact that $d v$ is pure imaginary to (3.18), we have

$$
d q^{*} \equiv \frac{1}{2 i(n+2)}\left\{-2 d v-2 i \operatorname{Im}\left(\frac{d f}{f}-v\right)(\xi) d \theta\right\} \quad(\bmod \theta)
$$

from which we obtain (3.10).

It determines a connection $-\eta$ of the complex line bundle $K_{M}$ to assign a 1-form $-\eta(\chi)$ to each frame $\chi$ of $K_{M}$ on each member $U$ of a family of open subsets such that $U$ 's cover $M$ and $-\eta(\chi)$ 's satisfy the transformation rule of connection forms on each intersection of the open sets. If a local frame is fixed, we shall often denote the connection form of $-\eta$ with respect to the fixed frame also by $-\eta$. The reason why we add minus sign is that we have the following example: Let $M$ be an $m$-dimensional manifold, $\kappa$ a linear connection and $\kappa(\chi)$ the connection form with respect to a local frame $\chi=\left(X_{1}, \ldots, X_{m}\right)$ of the tangent bundle $T M$. Then $\kappa$ naturally induces a connection of the line bundle $\bigwedge^{m} T^{*} M$, which is determined by each local 1-form $-\operatorname{trace}(\kappa(\chi))$ with respect to $\theta^{1} \wedge \cdots \wedge \theta^{m}$, where $\left\{\theta^{i}\right\}$ is the dual frame of $\chi$.

Let $\zeta$ be a nonvanishing global cross section of $K_{M}$. For each local frame $\chi$ of $K_{M}$, we define a local function $f_{\chi}$ by $\zeta=f_{\chi}$. Then we assign a local 1 -form

$$
-\frac{d f_{\chi}}{f_{\chi}}
$$

to each local frame $\chi$ of $K_{M}$, which gives us a connection of $K_{M}$. Writing this connection as $-\eta$, we see that the local 1 -form $-d f / f$ appearing in (3.11) is the connection form of $-\eta$ with respect to the local frame $\theta \wedge \theta^{1} \wedge \cdots \wedge \theta^{n}$. Also noting that the curvature form of a connection of $K_{M}$ is globally defined, we see that the condition $d(d f / f)=0$ implies that the curvature of the connection $-\eta$ defined by (3.21) is identically zero.

Let $(\phi, \xi, \theta)$ be a $\mathscr{D}$-preserving almost contact structure on $M, \nabla$ the TanakaWebster connection on $M$ associated with $(\phi, \xi, \theta)$ and $v_{\beta}{ }^{\gamma}$ the connection form of $\nabla$ with respect to a frame (1.6) on an open set $U$. Assign a local 1 -form $-v_{\alpha}{ }^{\alpha}=$ $-\operatorname{trace}\left(v_{\beta}^{\gamma}\right)$ to each frame $\theta \wedge \theta^{1} \wedge \cdots \wedge \theta^{n}$ of $K_{M}$ on $U$. Taking any two frames (1.6) and (3.9), we have

$$
\tilde{v}_{\beta}^{\gamma}=\left(X^{-1}\right)_{\alpha}^{\gamma} d X_{\beta}^{\alpha}+\left(X^{-1}\right)_{\lambda}^{\gamma} v_{\alpha}^{\lambda} X_{\beta}^{\alpha}
$$


on $U \cap \tilde{U}$, so that we have

$$
-\tilde{v}_{\alpha}{ }^{\alpha}=\operatorname{det} X d \operatorname{det}\left(X^{-1}\right)+\left(-v_{\alpha}{ }^{\alpha}\right)
$$

on $U \cap \tilde{U}$, where $\tilde{v}_{\beta}^{\gamma}$ is the connection form of $\nabla$ with respect to (3.9). Noting that $\theta \wedge \tilde{\theta}^{1} \wedge \cdots \wedge \tilde{\theta}^{n}=\operatorname{det}\left(X^{-1}\right) \theta \wedge \theta^{1} \wedge \cdots \wedge \theta^{n}$, we see that the system of the local 1-forms $-v_{\alpha}{ }^{\alpha}$ satisfies the transformation rule of connection forms of $K_{M}$. Therefore we have a connection of $K_{M}$. This connection of $K_{M}$ is called the connection induced by a Tanaka-Webster connection $\nabla$ and written as $-v$.

Considering a connection of $K_{M}$ instead of a global cross section of $K_{M}$ and taking (3.11) into account, we can obtain the following result for the case of CR Einstein-Weyl structures.

THEOREM 3.3. Let $(M, \mathscr{D}, J)$ be an orientable nondegenerate $C R$ manifold of dimension $2 n+1(n \geq 1)$. Assume that, for a $\mathscr{D}$-preserving almost contact structure $(\phi, \xi, \theta)$ on $M$, there is a connection $-\eta$ of $K_{M}$ such that

$$
\begin{gathered}
-d \eta\left(Y_{1}, \bar{Y}_{2}\right)=0, \\
u\left(\bar{Y}_{1}\right)=0
\end{gathered}
$$

for any $Y_{1}, Y_{2} \in \mathscr{D}^{1,0}$, where $-d \eta$ is the curvature form of the connection $-\eta$ and $u$ is the difference between the connections $-v$ and $-\eta$ defined by $u=$ $-v(\chi)-(-\eta(\chi))$ for an arbitrary local frame $\chi$ of $K_{M}$. Define a real valued 1form $p$ on $M$ by

$$
p=\frac{1}{n+2} \operatorname{Re}(u)
$$

Then the CR Weyl connection determined by $p$ and the almost contact structure $(\phi, \xi, \theta)$ on $M$ (cf. Corollary 2.3) is CR Einstein-Weyl.

Proof. Since the 2-form $d v_{\alpha}{ }^{\alpha}$ is pure imaginary, we have from (3.23)

$$
d p\left(Y_{1}, \bar{Y}_{2}\right)=\frac{1}{n+2} \operatorname{Re}(d \eta)\left(Y_{1}, \bar{Y}_{2}\right)=0
$$

for all $Y_{1}, Y_{2} \in \mathscr{D}^{1,0}$, which indicates that the 1-form $p$ satisfies (2.2). Therefore we see from Corollary 2.3 that the 1 -form $p$ and the $\mathscr{D}$-preserving almost contact structure $(\phi, \xi, \theta)$ determine a CR Weyl connection, which is denoted by $D$.

Take an arbitrary local frame (1.6) and its dual frame (1.7) with respect to $(\phi, \xi, \theta)$ on an open subset $U$. To show that $D$ is CR Einstein-Weyl, we see from Corollay 2.5 that it is sufficient to show there is a function $\Lambda$ such that

$$
i d v-(n+2) d q \equiv \Lambda d \theta \quad\left(\bmod \theta^{\beta} \wedge \theta^{\gamma}, \theta^{\bar{\beta}} \wedge \theta^{\bar{\gamma}}, \theta\right),
$$

where $-v$ simply denotes the connection form $-v\left(\theta \wedge \theta^{1} \wedge \cdots \wedge \theta^{n}\right)$ and $q=$ $-p \circ \phi$.

From the definition of $p$ and (3.12), we have 


$$
q=\frac{1}{n+2} \operatorname{Im}\left\{\left(\eta_{\beta}-v_{\beta}\right) \theta^{\beta}-\left(\eta_{\bar{\beta}}-v_{\bar{\beta}}\right) \theta^{\bar{\beta}}\right\}
$$

where $\eta_{1}=\eta_{\beta} \theta^{\beta}+\eta_{\bar{\beta}} \theta^{\bar{\beta}}+\eta_{0} \theta$ and $-\eta$ simply denotes the connection form $-\eta\left(\theta \wedge \theta^{1} \wedge \cdots \wedge \theta^{n}\right)$. Since

$$
\begin{aligned}
d\left\{\left(\eta_{\bar{\beta}}-v_{\bar{\beta}}\right) \theta^{\bar{\beta}}\right\}= & \left(\eta_{\bar{\beta} \bar{\gamma}}-v_{\bar{\beta} \bar{\gamma}}\right) \theta^{\gamma} \wedge \theta^{\bar{\beta}}+\left(\eta_{\bar{\beta} \bar{\gamma}}-v_{\bar{\beta} \bar{\gamma}}\right) \theta^{\bar{\gamma}} \wedge \theta^{\bar{\beta}} \\
& -\left(\eta_{\bar{\beta} 0}-v_{\bar{\beta} 0}\right) \theta^{\bar{\beta}} \wedge \theta-\left(\eta_{\bar{\gamma}}-v_{\bar{\gamma}}\right) A^{\bar{\gamma}} \theta^{\beta} \wedge \theta
\end{aligned}
$$

and $d\left\{\left(\eta_{\beta}-v_{\beta}\right) \theta^{\beta}\right\}$ is similarly calculated, we have

$$
\begin{aligned}
d q \equiv & \frac{1}{2 i(n+2)}\left\{-\eta_{\beta \bar{\gamma}}-\eta_{\bar{\gamma} \beta}+v_{\beta \bar{\gamma}}+v_{\bar{\gamma} \beta}-\overline{\eta_{\gamma \bar{\beta}}}-\overline{\eta_{\bar{\beta} \gamma}}+\overline{v_{\gamma \bar{\beta}}}+\overline{v_{\bar{\beta} \gamma}}\right\} \theta^{\beta} \wedge \theta^{\bar{\gamma}} \\
& \left(\bmod \theta^{\beta} \wedge \theta^{\gamma}, \theta^{\bar{\beta}} \wedge \theta^{\bar{\gamma}}, \theta\right) .
\end{aligned}
$$

On the other hand, from (3.23) and (1.22), we have

$$
-\eta_{\beta \bar{\gamma}}+\eta_{\bar{\gamma} \beta}+i g_{\beta \bar{\gamma}} \eta_{0}=0 .
$$

Also, from (3.24), we have $\left(\eta_{\bar{\beta}}-v_{\bar{\beta}}\right) \theta^{\bar{\beta}} \equiv 0$. Therefore the right hand side of (3.28) identically vanishes. In particular we have

$$
\eta_{\bar{\beta} \gamma}-v_{\bar{\beta} \gamma}=0 \text {. }
$$

Applying (3.30) and (3.31) to (3.29), we have

$$
\begin{aligned}
d q \equiv & \frac{1}{2 i(n+2)}\left\{v_{\beta \bar{\gamma}}-v_{\bar{\gamma} \beta}+\overline{v_{\gamma \bar{\beta}}}-\overline{v_{\bar{\beta} \gamma}}-2 i \operatorname{Im}\left(\eta_{0}\right) i g_{\beta \bar{\gamma}}\right\} \theta^{\beta} \wedge \theta^{\bar{\gamma}} \\
& \left(\bmod \theta^{\beta} \wedge \theta^{\gamma}, \theta^{\bar{\beta}} \wedge \theta^{\bar{\gamma}}, \theta\right) .
\end{aligned}
$$

Moreover, applying (3.19) and the fact that $d v$ is pure imaginary to (3.32), we have

$$
d q \equiv \frac{1}{2 i(n+2)}\{-2 d v-2 i \operatorname{Im}(u)(\xi) d \theta\} \quad\left(\bmod \theta^{\beta} \wedge \theta^{\gamma}, \theta^{\bar{\beta}} \wedge \theta^{\bar{\gamma}}, \theta\right)
$$

from which we obtain (3.26).

\section{CR Weyl structures and connections of the canonical bundle}

In this section, we consider a nondegenerate $\mathrm{CR}$ manifold which is not necessarily orientable. Thus $\mathscr{D}$-preserving almost contact structures are defined on open subsets which cover $M$. Turning our attention to the 1-form (3.25), we shall obtain the following Theorem 4.1. To begin with we prepare the following result.

Let $K_{U}$ be the restricted line bundle of $K_{M}$ to an open set $U$. Take any two $\mathscr{D}$-preserving almost contact structures $(U,(\phi, \xi, \theta))$ and $\left(U^{\prime},\left(\phi^{\prime}, \xi^{\prime}, \theta^{\prime}\right)\right)$. Let $\nabla$ 
(resp. $\left.\nabla^{\prime}\right)$ be the Tanaka-Webster connection associated with $(U,(\phi, \xi, \theta))$ (resp. $\left.\left(U^{\prime},\left(\phi^{\prime}, \xi^{\prime}, \theta^{\prime}\right)\right)\right)$ and $-v\left(\right.$ resp. $\left.-v^{\prime}\right)$ the connection of $K_{U}\left(\right.$ resp. $\left.K_{U^{\prime}}\right)$ induced by $\nabla\left(\right.$ resp. $\left.\nabla^{\prime}\right)$. We investigate the relation between $-v$ and $-v^{\prime}$. Take arbitrary admissible frames $(1.6)$ and $(1.17)$ of $(U,(\phi, \xi, \theta))$ and $\left(U^{\prime},\left(\phi^{\prime}, \xi^{\prime}, \theta^{\prime}\right)\right)$ respectively such that $Z_{\alpha}=Z_{\alpha}^{\prime}$ and (1.19) hold for any indices $\alpha$ on $U \cap U^{\prime}$. From the definition of connections induced by Tanaka-Webster connections, we have

$$
\begin{gathered}
-v\left(\theta \wedge \theta^{1} \wedge \cdots \wedge \theta^{n}\right)=-\operatorname{trace}\left(v_{\beta}{ }^{\gamma}\right)=-v_{\alpha}{ }^{\alpha}, \\
-v^{\prime}\left(\theta^{\prime} \wedge{ }^{\prime} \theta^{1} \wedge \cdots \wedge^{\prime} \theta^{n}\right)=-\operatorname{trace}\left({ }^{\prime} v_{\beta}{ }^{\gamma}\right)=-{ }^{\prime} v_{\alpha}{ }^{\alpha},
\end{gathered}
$$

where $v_{\beta}{ }^{\gamma}$ (resp. ' $v_{\beta}{ }^{\gamma}$ ) is the connection form of $\nabla$ (resp. $\nabla^{\prime}$ ) with respect to (1.6) (resp. (1.17)). From (1.24), we have

$$
\begin{aligned}
-^{\prime} v_{\alpha}{ }^{\alpha}= & -v_{\alpha}{ }^{\alpha}-2(n+1) \mu_{\alpha} \theta^{\alpha}+2 \mu_{\bar{\alpha}} \theta^{\bar{\alpha}} \\
& -i\left\{\mu_{\alpha}^{\alpha}+\mu_{\alpha}{ }^{\alpha}+4(n+1) \mu_{\alpha} \mu^{\alpha}-i n \mu_{0}\right\} \theta
\end{aligned}
$$

on $U \cap U^{\prime}$. On the other hand, since

$$
\theta^{\prime} \wedge^{\prime} \theta^{1} \wedge \cdots \wedge^{\prime} \theta^{n}=\varepsilon e^{2 \mu} \theta \wedge \theta^{1} \wedge \cdots \wedge \theta^{n}
$$

we have

$$
-v\left(\theta^{\prime} \wedge^{\prime} \theta^{1} \wedge \cdots \wedge^{\prime} \theta^{n}\right)=2 d \mu+\left(-v_{\alpha}^{\alpha}\right) .
$$

Then from (4.1) and (4.3), we have

$$
\begin{aligned}
-v^{\prime}-(-v)= & -2(n+2) \mu_{\alpha} \theta^{\alpha}-(n+2) \mu_{0} \theta-i\left\{\mu_{\alpha}^{\alpha}+\mu_{\alpha}{ }^{\alpha}+4(n+1) \mu_{\alpha} \mu^{\alpha}\right\} \theta \\
= & -(n+2)\left(\mu_{\alpha} \theta^{\alpha}+\mu_{\bar{\alpha}} \theta^{\bar{\alpha}}+\mu_{0} \theta\right)-(n+2)\left(\mu_{\alpha} \theta^{\alpha}-\mu_{\bar{\alpha}} \theta^{\bar{\alpha}}\right) \\
& -i\left\{\mu^{\alpha}{ }_{\alpha}+\mu_{\alpha}{ }^{\alpha}+4(n+1) \mu_{\alpha} \mu^{\alpha}\right\} \theta \\
= & -(n+2) d \mu-i(n+2) q^{*}-i\left\{\operatorname{trace}_{\mathscr{D}} \nabla P^{*}+2(n+1) p^{*}\left(P^{*}\right)\right\} \theta
\end{aligned}
$$

where $-v^{\prime}-(-v)$ is the difference between the connections $-v^{\prime}$ and $-v$ and we have used (1.15), (1.16) and (1.33).

Let $\mathscr{A}$ be the set of all connections of $K_{M}$ satisfying

$$
\operatorname{Re}(-d \eta)\left(Y_{1}, \bar{Y}_{2}\right)=0
$$

for all $Y_{1}, Y_{2} \in \mathscr{D}^{1,0}$, where $-d \eta$ denotes the curvature form of the connection $-\eta$ and we note that $-d \eta$ is globally defined on $M$. We define an equivalence relation in the set $\mathscr{A}$ by setting $-\eta \sim-\kappa$ if and only if the difference $-\eta-(-\kappa)$ is a pure imaginary 1 -form on $M$. And we write the set of all equivalence classes as $\mathscr{A} / \sim$. Let $\mathscr{B}$ be the set of all CR Weyl connections on $M$. Then we obtain

THEOREM 4.1. There exists a one to one correspondence between $\mathscr{A} / \sim$ and $\mathscr{B}$.

Proof. To begin with, note that a connection $-\eta$ of $K_{M}$ induces a connection of $K_{U}$ for an arbitrary open set $U$. The induced connection of $K_{U}$ is denoted also by $-\eta$. 
Take a connection $-\eta$ of $K_{M}$ in the set $\mathscr{A}$. For each $\mathscr{D}$-preserving almost contact structure $(U,(\phi, \xi, \theta))$, we have the difference $u$ between $-v$ and $-\eta$, where $-v$ is the connection of $K_{U}$ induced by the Tanaka-Webster connection associated with $(U,(\phi, \xi, \theta))$. Note that $u$ is a complex valued 1-form on $U$. Define a real valued 1 -form $p$ on $U$ by (3.25). For any two $\mathscr{D}$-preserving almost contact structures $(U,(\phi, \xi, \theta))$ and $\left(U^{\prime},\left(\phi^{\prime}, \xi^{\prime}, \theta^{\prime}\right)\right)$, from (4.4), we have

$$
\begin{aligned}
u^{\prime}= & -v^{\prime}-(-\eta) \\
= & -(-\eta)-v-(n+2) d \mu-i(n+2) q^{*} \\
& -i\left\{\operatorname{trace}_{\mathscr{D}} \nabla P^{*}+2(n+1) p^{*}\left(P^{*}\right)\right\} \theta \\
= & u-(n+2) d \mu-i(n+2) q^{*}-i\left\{\operatorname{trace}_{\mathscr{D}} \nabla P^{*}+2(n+1) p^{*}\left(P^{*}\right)\right\} \theta,
\end{aligned}
$$

where $u^{\prime}$ is the difference between $-v^{\prime}$ and $-\eta$. From (3.25) and (4.6), we obtain

$$
p^{\prime}=p-d \mu \text { on } U \cap U^{\prime} .
$$

Noting that each 1-form $p$ satisfies (2.2) by (4.5), this system of local 1-forms determines a CR Weyl connection $D_{\eta}$ by virtue of Lemma 2.2. We can define a map $w: \mathscr{A} \rightarrow \mathscr{B}$ by $-\eta \mapsto D_{\eta}$.

For two connections $-\eta$ and $-\kappa$ of $K_{M}$ contained in the set $\mathscr{A}$, assume that $D_{\eta}=D_{\kappa}$. The associated local 1-forms of $D_{\eta}$ and $D_{\kappa}$ relative to the same $\mathscr{D}$ preserving almost contact structure $(U,(\phi, \xi, \theta))$ coincide. Thus we have

$$
\operatorname{Re}(\eta(\chi))=\operatorname{Re}(\kappa(\chi))
$$

where $\chi$ is an arbitrary frame of $K_{M}$ on $U$. The equation (4.7) states that the difference between $-\eta$ and $-\kappa$ is a pure imaginary 1 -form on $M$. Therefore the map $\mathscr{A} / \sim \rightarrow \mathscr{B}$ induced by $w$ is injective.

We show that $w$ is surjective to complete the proof. Fix an arbitrary CR Weyl connection $D$. The CR Weyl connection $D$ assigns the associated 1form $p$ on $U$ which satisfies (2.2) to each $\mathscr{D}$-preserving almost contact structure $(U,(\phi, \xi, \theta))$. Note that the system of these 1 -forms satisfies the property (2.3). For a frame $\chi$ of $K_{U}$, we define a 1 -form $-\kappa_{0}(\chi)$ on $U$ by

$$
-\kappa_{0}(\chi)=-(n+2) p-i(n+2)\left\{q+p(P) \theta+\frac{1}{4(n+1)(n+2)} \rho^{\nabla} \theta\right\}-v(\chi),
$$

where $\rho^{\nabla}$ is the scalar curvature of the Tanaka-Webster connection $\nabla$ associated with $(U,(\phi, \xi, \theta))$. Note that the 1 -form in the right hand side of (4.8)

$$
-(n+2) p-i(n+2)\left\{q+p(P) \theta+\frac{1}{4(n+1)(n+2)} \rho^{\nabla} \theta\right\}
$$

is independent of the choice of the local frame $\chi$ of $K_{U}$. Thus the system of the local 1-forms $-\kappa_{0}(\chi)$ defined by (4.8) determines a connection of $K_{U}$.

If, for any two $\mathscr{D}$-preserving almost contact structures $(U,(\phi, \xi, \theta))$ and $\left(U^{\prime},\left(\phi^{\prime}, \xi^{\prime}, \theta^{\prime}\right)\right)$, the connection forms $-\kappa_{0}^{\prime}(\chi)$ of $K_{U^{\prime}}$ and $-\kappa_{0}(\chi)$ of $K_{U}$ with 
respect to an arbitrary local frame $\chi$ on $U \cap U^{\prime}$ coincide, we see that the family of the locally defined connections determines a connection of $K_{M}$. From (2.3), (2.4) and (2.10), we have

$$
\left(q^{\prime}+p^{\prime}\left(P^{\prime}\right) \theta^{\prime}\right)-(q+p(P) \theta)=-\left(q^{*}+p^{*}\left(P^{*}\right) \theta\right)
$$

on $U \cap U^{\prime}$. Thus, applying (1.32), (4.4) and (4.9) to (4.8), we have $-\kappa_{0}^{\prime}(\chi)=$ $-\kappa(\chi)$ for every frame $\chi$ on $U \cap U^{\prime}$. Thus (4.8) determines a connection $-\kappa_{0}$ of $K_{M}$. Since $D=D_{\kappa_{0}}$ by (4.8), the map $w$ is surjective.

From Theorem 3.3 and Theorem 4.1, we immediately obtain

Corollary 4.2. Let $(M, \mathscr{D}, J)$ be a nondegenerate $C R$ manifold of dimension $2 n+1(n \geq 1)$. If a connection $-\eta$ of $K_{M}$ satisfies (3.23) and for each $\mathscr{D}$-preserving almost contact structure $(U,(\phi, \xi, \theta))$, the difference $u$ between $-v$ and $-\eta$ satisfies (3.24), the CR Weyl connection $D_{\eta}$ corresponding to $-\eta$ is $C R$ Einstein-Weyl.

Remark. For each $\mathscr{D}$-preserving almost contact structure $(U,(\phi, \xi, \theta))$, the difference $u$ between $-v$ and $-\eta$ is a complex valued 1 -form on $U$. For any two $\mathscr{D}$-preserving almost contact structures $(U,(\phi, \xi, \theta))$ and $\left(U^{\prime},\left(\phi^{\prime}, \xi^{\prime}, \theta^{\prime}\right)\right)$, we have from (4.4)

$$
u^{\prime}-u \equiv-(n+2)\left(p^{*}+i q^{*}\right) \quad(\bmod \theta)
$$

on $U \cap U^{\prime}$. Since the local 1 -form $p^{*}+i q^{*}$ is a $(1,0)$-form, the equation (3.24) is a global condition on $M$. Therefore, if the condition (3.24) holds for a connection $-\eta$, we say that $-\eta$ or $D_{\eta}$ satisfies (3.24).

Moreover, we have from Theorem 3.3 the following proposition.

Proposition 4.3. $(M, \mathscr{D}, J)$ admits a CR Einstein-Weyl connection if and only if there exists a connection of $K_{M}$ satisfying (3.23) and (3.24) for $(\mathscr{D}, J)$.

Proof. Since we have shown the if part in Corollary 4.2, we shall show that if there exists a CR Einstein-Weyl connection on $M$, there exists a connection of $K_{M}$ satisfying (3.23) and (3.24).

Let $D$ be a CR Einstein-Weyl connection on $M$. Consider the connection $-\kappa_{0}$ of $K_{M}$ defined by (4.8). We note that, for each $\mathscr{D}$-preserving almost contact structure $(U,(\phi, \xi, \theta))$, the difference $u$ between $-v$ and $-\kappa_{0}$ satisfies (3.24).

Since $D$ is a CR Einstein-Weyl connection, it satisfies the equation (2.22). For each almost contact structure $(U,(\phi, \xi, \theta))$, from (1.28) and (2.30), we have

$$
\begin{aligned}
& d v\left(Y_{1}, \bar{Y}_{2}\right)+i(n+2) d q\left(Y_{1}, \bar{Y}_{2}\right) \\
& \quad=2 i(n+2)\left\{\lambda-\frac{1}{2} p(P)-\frac{1}{8(n+1)(n+2)} \rho^{\nabla}\right\} d \theta\left(Y_{1}, \bar{Y}_{2}\right)
\end{aligned}
$$


for any $Y_{1}, Y_{2} \in \mathscr{D}^{1,0}$, where $-d v$ is the curvature form of the connection $-v$ of $K_{U}$ and $\lambda$ is a function defined on $U$. Furthermore, from (4.8), we have

$$
\begin{aligned}
-d \kappa_{0}\left(Y_{1}, \bar{Y}_{2}\right)= & -d v\left(Y_{1}, \bar{Y}_{2}\right)-i(n+2) d q\left(Y_{1}, \bar{Y}_{2}\right) \\
& -i(n+2)\left\{p(P)+\frac{1}{4(n+1)(n+2)} \rho^{\nabla}\right\} d \theta\left(Y_{1}, \bar{Y}_{2}\right)
\end{aligned}
$$

for any $Y_{1}, Y_{2} \in \mathscr{D}^{1,0}$, where $-d \kappa_{0}$ is the curvature form of the connection $-\kappa_{0}$ of $K_{M}$ and we have used the fact that each associated 1-form $p$ of $D$ relative to $(U,(\phi, \xi, \theta))$ satisfies (2.2). Substituting (4.10) into (4.11), we have

$$
-d \kappa_{0}\left(Y_{1}, \bar{Y}_{2}\right)=-2 i(n+2) \lambda d \theta\left(Y_{1}, \bar{Y}_{2}\right)
$$

for any $Y_{1}, Y_{2} \in \mathscr{D}^{1,0}$. Define a 1 -form $-\eta$ by

$$
-\eta=-\kappa_{0}+2 i(n+2) \lambda \theta .
$$

Since the system of the functions $\lambda$ satisfies (2.23), we see that $\lambda \theta$ is a global 1 -form on $M$. Therefore $-\eta$ becomes a connection of $K_{M}$. From (4.12) and (4.13), we have

$$
-d \eta\left(Y_{1}, \bar{Y}_{2}\right)=0
$$

for all $Y_{1}, Y_{2} \in \mathscr{D}^{1,0}$. Therefore the connection $-\eta$ of $K_{M}$ satisfies (3.23) and (3.24).

Remark. Since the 1 -form $2 i(n+2) \lambda \theta$ on $M$ is pure imaginary, we obtain $D_{\kappa_{0}}=D_{\eta}$ by Theorem 4.1 .

Let $\{(U,(\phi, \xi, \theta))\}$ be a family of $\mathscr{D}$-preserving almost contact structures on open sets $U$ which cover $M$. Let $\mathscr{A}^{*}$ be the set of all connections $-\kappa$ of $K_{M}$ satisfying (3.24) and, for each $(U,(\phi, \xi, \theta))$,

$$
-d \kappa\left(Y_{1}, \bar{Y}_{2}\right)=-i \lambda d \theta\left(Y_{1}, \bar{Y}_{2}\right)
$$

for some real valued function $\lambda$ on $U$. We define an equivalence relation on the set $\mathscr{A}^{*}$ by setting $-\kappa \sim-\vartheta$ if and only if there is a system of real valued functions $a$ such that $-\kappa-(-\vartheta)=i a \theta$ and $a^{\prime}=\varepsilon e^{-2 \mu} a$ on each the nonempty intersection $U \cap U^{\prime}$. And we write the set of all equivalence classes as $\mathscr{A}^{*} / \sim$. Moreover, let $\mathscr{B}^{*}$ be the set of all CR Einstein-Weyl connections on $M$. Then from the proof of Proposition 4.3, we also have the following corollary:

COROLlaRy 4.4. There exists a one to one correspondence between $\mathscr{A}^{*} / \sim$ and $\mathscr{B}^{*}$.

Remark. Since $-d \kappa$ is a global 2-form on $M$, for any two $\mathscr{D}$-preserving almost contact structures $(U,(\phi, \xi, \theta))$ and $\left(U^{\prime},\left(\phi^{\prime}, \xi^{\prime}, \theta^{\prime}\right)\right)$, we have $\lambda^{\prime}=\varepsilon e^{-2 \mu} \lambda$ on $U \cap U^{\prime}$. 
Proof. Let $-\kappa$ be a connection of $K_{M}$ satisfying (3.24) and (4.15). Take an admissible frame (1.6) and the dual frame $(1.7)$ of $(U,(\phi, \xi, \theta))$. From (4.15), we have

$$
-d \kappa \equiv \lambda g_{\beta \bar{\gamma}} \theta^{\beta} \wedge \theta^{\bar{\gamma}} \quad\left(\bmod \theta^{\beta} \wedge \theta^{\gamma}, \theta^{\bar{\beta}} \wedge \theta^{\bar{\gamma}}, \theta\right)
$$

on $U$. Thus, since

$$
\begin{aligned}
& \operatorname{Re}(-d \kappa) \equiv \frac{1}{2}\left\{\lambda g_{\beta \bar{\gamma}} \theta^{\beta} \wedge \theta^{\bar{\gamma}}+\overline{\lambda g_{\beta \bar{\gamma}} \theta^{\beta} \wedge \theta^{\bar{\gamma}}}\right\} \quad\left(\bmod \theta^{\beta} \wedge \theta^{\gamma}, \theta^{\bar{\beta}} \wedge \theta^{\bar{\gamma}}, \theta\right) \\
& \equiv \frac{1}{2}\left(\lambda g_{\beta \bar{\gamma}}-\lambda \overline{g_{\gamma \bar{\beta}}}\right) \theta^{\beta} \wedge \theta^{\bar{\gamma}} \quad\left(\bmod \theta^{\beta} \wedge \theta^{\gamma}, \theta^{\bar{\beta}} \wedge \theta^{\bar{\gamma}}, \theta\right) \\
& \equiv 0 \quad\left(\bmod \theta^{\beta} \wedge \theta^{\gamma}, \theta^{\bar{\beta}} \wedge \theta^{\bar{\gamma}}, \theta\right),
\end{aligned}
$$

we see that the set $\mathscr{A}^{*}$ is contained in the set $\mathscr{A}$. Therefore we may restrict the domain of the map $w$ to the set $\mathscr{A}^{*}$. We denote this restricted map also by $w$ and put $w(-\kappa)=D_{\kappa}$ for every $-\kappa \in \mathscr{A}^{*}$.

Take a connection $-\kappa$ in $\mathscr{A}^{*}$. Define a 1 -form $-\eta$ by

$$
-\eta=-\kappa+i \lambda \theta
$$

on each $U$. Then $-\eta$ becomes a connection of $K_{M}$ since $\lambda \theta$ is a global 1-form on $M$. Since this connection $-\eta$ of $K_{M}$ satisfies (3.23) and (3.24), the CR Weyl connection $D_{\eta}$ is CR Einstein-Weyl in virtue of Corollary 4.2. Also from (4.16) and Theorem 4.1, we have $D_{\kappa}=D_{\eta}$. Therefore the image of the map $w$ is contained in the set $\mathscr{B}^{*}$.

For two connections $-\kappa$ and $-\vartheta$ of $K_{M}$ contained in the set $\mathscr{A}^{*}$, assume that $D_{\kappa}=D_{\vartheta}$. From Theorem 4.1, there is a pure imaginary 1-form $\alpha$ on $M$ such that $-\kappa-(-\mathscr{Y})=\alpha$. From (3.24), we have $\alpha\left(\bar{Y}_{1}\right)=0$ for every $Y_{1} \in \mathscr{D}^{1,0}$, which also implies that $\alpha\left(Y_{1}\right)=0$ for every $Y_{1} \in \mathscr{D}^{1,0}$, because $\alpha$ is pure imaginary. Thus there is a system of real valued functions $a$ such that $\alpha=i a \theta$ on $U$. Therefore the map $\mathscr{A}^{*} / \sim \rightarrow \mathscr{B}^{*}$ induced by $w$ is injective.

Fix a CR Einstein-Weyl connection $D$ on $M$. For this connection $D$, define the connection $-\kappa_{0}$ of $K_{M}$ by (4.8). From the poof of Proposition 4.3, this connection $-\kappa_{0}$ is contained in the set $\mathscr{A}^{*}$ and satisfies $D=D_{\kappa_{0}}$. Therefore the map $\mathscr{A}^{*} / \sim \rightarrow \mathscr{B}^{*}$ is surjective.

\section{Example of CR Einstein-Weyl real hypersurfaces in complex manifolds}

Let $N$ be a complex manifold of complex dimension $n+1$ and $J$ the almost complex structure naturally induced by the complex structure on $N$. Also let $\imath: M \rightarrow N$ be an imbedded real hypersurface in $N$. We often omit the notation $l_{*}\left(\right.$ resp. $\left.l^{*}\right)$ which denotes the differential map (resp. the pullback) of $\imath$ throughout this section. We define $\mathscr{D}$ by

$$
\mathscr{D}=T M \cap J T M .
$$


Then $\mathscr{D}$ becomes a real vector bundle of rank $2 n$. The almost complex structure $J$ induces a complex structure on $\mathscr{D}$, which is denoted also by $J$. Let $\mathscr{D}^{1,0}$ be a subbundle of $\mathbf{C} \mathscr{D}$ composed of the eigenvectors corresponding to $i$ of $J$. Then since we have

$$
\mathscr{D}^{1,0}=T^{1,0} N \cap \mathbf{C T M},
$$

we see that $\mathscr{D}^{1,0}$ satisfies the integrability condition $(\mathrm{C})$ in Section 1 , where $T^{1,0} N$ the subbundle of CTN consisting of all complexified tangent vectors of type $(1,0)$. Thus the pair $(\mathscr{D}, J)$ on $M$ becomes a CR structure on $M$, which is called an induced $C R$ structure on $M$. A real hypersurface $M$ in the complex manifold $N$ is nondegenerate if the induced CR structure on $M$ is nondegenerate. We shall consider only the nondegenerate real hypersurface $M$ in $N$.

Let $K_{N}$ be the holomorphic line bundle consisting of all $(n+1,0)$-forms on $N$, that is, $K_{N}=\bigwedge^{n+1} T_{1,0} N$, where $T_{1,0} N$ is the dual bundle of $T^{1,0} N . \quad K_{N}$ is called the canonical line bundle on $N$. We denote a connection of a line bundle by $-\eta$ and the connection form with respect to a local frame $\chi$ by $-\eta(\chi)$ as we did so in Section 3. Let $-\eta$ be a connection of $K_{N}$. If $\chi$ is a local frame of $K_{N}$, that is, $\chi$ is a nonvanishing local $(n+1,0)$-form on $N$, the pullback of $\chi$ is a local frame of $K_{M}$, which is denoted also by $\chi$, where $K_{M}$ is the canonical bundle on $M$. Thus assigning the local frame $\chi$ of $K_{N}$ a local 1-form $-l^{*}(\eta(\chi))$, we obtain a connection of $K_{M}$ which is called the induced connection of $K_{M}$ and denoted by $-\eta^{*}$, that is,

$$
-\eta^{*}(\chi)=-l^{*}(\eta(\chi))
$$

In general, a connection of a holomorphic vector bundle is holomorphic if the connection form with respect to every holomorphic local frame is a holomorphic 1-form. From Proposition 4.3, we obtain

THEOREM 5.1. Let $N$ be a complex manifold with a holomorphic connection of $K_{N}$. Then every nondegenerate real hypersurface $M$ in $N$ admits a CR EinsteinWeyl structure.

Proof. Fix an arbitrary $\mathscr{D}$-preserving almost contact structure $(U,(\phi, \xi, \theta))$. Let $\nabla$ be the Tanaka-Webster connection associated with $(U,(\phi, \xi, \theta))$ and $-v$ the connection of $K_{U}$ induced by $\nabla$. On each $U$, we can take a frame of $K_{U}$ as

$$
\imath^{*}\left(d z^{1} \wedge \cdots \wedge d z^{n+1}\right)
$$

if necessary we shrink $U$, where $\left(z^{1}, \ldots, z^{n+1}\right)$ is a local complex coordinate on $N$. To simplify the notation, we also denote (5.2) by $d z^{1} \wedge \cdots \wedge d z^{n+1}$. Taking an admissible frame (1.6) and the dual frame (1.7) on $U$, we obtain

$$
\begin{aligned}
-v\left(d z^{1} \wedge \cdots \wedge d z^{n+1}\right) & =\frac{d f}{f}+\left\{-v\left(\theta \wedge \theta^{1} \wedge \cdots \wedge \theta^{n}\right)\right\} \\
& =\frac{d f}{f}-v_{\alpha}{ }^{\alpha}
\end{aligned}
$$


where $v_{\alpha}^{\alpha}=\operatorname{trace}\left(v_{\beta}{ }^{\gamma}\right), v_{\beta}{ }^{\gamma}$ is the connection form of $\nabla$ with respect to (1.6) and $f$ is the complex valued function defined by

$$
d z^{1} \wedge \cdots \wedge d z^{n+1}=f \theta \wedge \theta^{1} \wedge \cdots \wedge \theta^{n}
$$

on $U$. Since the $(n+1,0)$-form (5.2) is a closed form, we have

$$
-v\left(d z^{1} \wedge \cdots \wedge d z^{n+1}\right)\left(\bar{Y}_{1}\right)=0
$$

for every $Y_{1} \in \mathscr{D}^{1,0}$ as in the proof of Lemma 3.2. Note that the equation (5.5) is independent of the choice of the local coordinates $\left(z^{1}, \ldots, z^{n+1}\right)$ on $N$. Therefore (5.5) is a property of the induced CR structure on a real hypersurface in a complex manifold.

Let $-\eta$ be a holomorphic connection of $K_{N}$. Since $d z^{1} \wedge \cdots \wedge d z^{n+1}$ is a holomorphic local frame of $K_{N},-\eta\left(d z^{1} \wedge \cdots \wedge d z^{n+1}\right)$ is a holomorphic 1-form. Thus we have

$$
-\eta^{*}\left(d z^{1} \wedge \cdots \wedge d z^{n+1}\right)\left(\bar{Y}_{1}\right)=0
$$

for every $Y_{1} \in \mathscr{D}^{1,0}$. Combining (5.5) with (5.6), we see that the induced connection $-\eta^{*}$ satisfies (3.24). On the other hand, for the curvature form of the induced connection $-\eta^{*}$, from (5.1), we have

$$
\begin{aligned}
-d \eta^{*}\left(Y_{1}, \bar{Y}_{2}\right) & =-d\left(\eta^{*}(\chi)\right)\left(Y_{1}, \bar{Y}_{2}\right) \\
& =-d(\eta(\chi))\left(l_{*} Y_{1}, l_{*} \bar{Y}_{2}\right) \\
& =0
\end{aligned}
$$

for all $Y_{1}, Y_{2} \in \mathscr{D}^{1,0}$, where $\chi$ is an arbitrary holomorphic local frame of $K_{N}$. Thus the induced connection $-\eta^{*}$ satisfies (3.23).

Therefore, from Corollary 4.2, the CR Weyl connection determined by the induced connection $-\eta^{*}$ of $K_{M}$ is CR Einstein-Weyl.

By making use of Theorem 5.1, we give some examples.

Example 1. Let $\imath: M \rightarrow N$ be a nondegenerate real hypersurface in $\mathbf{C}^{n+1}$. Assign zero-form on $\mathbf{C}^{n+1}$ to the global holomorphic frame $d z^{1} \wedge \cdots \wedge d z^{n+1}$ of $K_{\mathbf{C}^{n+1}}$ derived from the standard coordinate $\left(z^{1}, \ldots, z^{n+1}\right)$ of $\mathbf{C}^{n+1}$. Then we have a flat holomorphic connection of $K_{N}$. Thus, by Theorem 5.1, we obtain a CR Einstein-Weyl connection on $M$, which is written as $D_{0}$. We investigate the CR Einstein-Weyl connection $D_{0}$ in detail. To begin with, note that the pullback of $d z^{1} \wedge \cdots \wedge d z^{n+1}$ is the global frame of $K_{M}$. Fix an arbitrary $\mathscr{D}$ preserving almost contact structure $(U,(\phi, \xi, \theta))$ and take an admissible frame (1.6) and the dual frame (1.7) on an open set $U$. Then $D_{0}$ is determined by the following 1 -form 


$$
\begin{aligned}
p & =\frac{1}{n+2} \operatorname{Re}\left\{0-v\left(d z^{1} \wedge \cdots \wedge d z^{n+1}\right)\right\} \\
& =\frac{1}{n+2} \operatorname{Re}\left\{\frac{d f}{f}-v\left(\theta \wedge \theta^{1} \wedge \cdots \wedge \theta^{n}\right)\right\} \\
& =\frac{1}{n+2} \operatorname{Re}\left(\frac{d f}{f}-v_{\alpha}^{\alpha}\right),
\end{aligned}
$$

where $v_{\alpha}{ }^{\alpha}=\operatorname{trace}\left(v_{\beta}{ }^{\gamma}\right), v_{\beta}{ }^{\gamma}$ is the connection form of $\nabla$ with respect to (1.6) and $f$ is the complex valued function defined by (5.4). From (3.8) and (3.11), the local 1-form $p$ is exact. Thus from the first remark in Section 2, there is a $\mathscr{D}$ preserving almost contact structure $\left(U^{\prime},\left(\phi^{\prime}, \xi^{\prime}, \theta^{\prime}\right)\right)$ such that $\nabla^{\prime}=D_{0}$ on $U^{\prime}$, where $\nabla^{\prime}$ is the Tanaka-Webster connection associated with $\left(U^{\prime},\left(\phi^{\prime}, \xi^{\prime}, \theta^{\prime}\right)\right)$. In fact $\theta^{\prime}=\varepsilon e^{2 \mu} \theta$ for the function $\mu$ defined by (3.8). Therefore, by Lemma 3.2, $\left(U^{\prime},\left(\phi^{\prime}, \xi^{\prime}, \theta^{\prime}\right)\right)$ coincides with the pseudo-Einstein structure obtained in Lee [7].

Example 2. Let $l: M \rightarrow N$ be a nondegenerate real hypersurface in $\mathbf{C}^{n+1}$. Assign a global 1-form

$$
\sum_{\beta, \gamma}^{n+1} c_{\beta \gamma} z^{\beta} d z^{\gamma}
$$

to the global holomorphic frame $d z^{1} \wedge \cdots \wedge d z^{n+1}$ of $K_{\mathbf{C}^{n+1}}$, where $\left(z^{1}, \ldots, z^{n+1}\right)$ is the standard coordinate of $\mathbf{C}^{n+1}$ and $c_{\beta \gamma}$ is constant. Then we have a holomorphic connection of $K_{N}$ whose curvature form is

$$
\sum_{\beta, \gamma}^{n+1} c_{\beta \gamma} d z^{\beta} \wedge d z^{\gamma} .
$$

This connection of $K_{N}$ induces a CR Einstein-Weyl connection on $M$.

Example 3. Let $(N, h)$ be a pair of a complex manifold $N$ of complex dimension $n+1$ and a Hermitian metric $h$ on $N$, that is, $h$ is a Riemannian metric satisfying

$$
h(J X, J Y)=h(X, Y)
$$

for all $X, Y \in T N$, where $J$ is the almost complex structure naturally induced by the complex structure on $N$.

Let $A^{p, q}$ be the module of the $(p, q)$-forms on $N$. Then the exterior differential $d \varphi$ of any form $\varphi \in A^{p, q}$ is the sum of a $(p+1, q)$-form and a $(p, q+1)$ form denoted respectively by $d^{\prime} \varphi$ and $d^{\prime \prime} \varphi$.

We uniquely extend $h$ to a complex bilinear form, denoted also by $h$. For all $Z, W \in T^{1,0} N$,

$$
(Z, W) \mapsto h(Z, \bar{W})
$$


is a sesquilinear form which becomes a hermitian fiber metric on the holomorphic vector bundle $T^{1,0} N$. Then there exists a unique connection $\mathcal{D}$ of $T^{1,0} N$ such that the connection form with respect to every holomorphic local frame is a $(1,0)$-form and the connection is compatible with $h$, that is, for any $Z, W \in \Gamma\left(T^{1,0} N\right)$,

$$
d(h(Z, \bar{W}))=h(\mathcal{D} Z, \bar{W})+h(Z, \overline{\mathcal{D} W}),
$$

which is called the Hermitian connection of $h$ (cf. [4]). When we denote the connection form of $\mathcal{D}$ with respect to a holomorphic local frame $\left(\partial / \partial z^{1}, \ldots\right.$, $\left.\partial / \partial z^{n+1}\right)$ by $\eta_{\beta}^{\gamma}$, that is, $\mathcal{D}\left(\partial / \partial z^{\beta}\right)=\eta_{\beta}{ }^{\gamma}\left(\partial / \partial z^{\gamma}\right)$, we have

$$
\eta_{\beta}^{\gamma}=d^{\prime} h_{\beta \bar{\alpha}} h^{\bar{\alpha} \gamma}
$$

where $h_{\beta \bar{\alpha}}=h\left(\partial / \partial z^{\beta}, \partial / \partial z^{\bar{\alpha}}\right)$ and $h^{\bar{\alpha} \gamma}$ is a component of the inverse matrix of the hermitian matrix $\left(h_{\beta \bar{\alpha}}\right)$. The Hermitian connection of $h$ naturally induces a connection of the canonical line bundle $K_{N}$, which is denoted by $-\eta$ as usual. In fact, the connection form of $-\eta$ with respect to the local holomorphic frame $d z^{1} \wedge \cdots \wedge d z^{n+1}$ is

(5.11) $-\eta\left(d z^{1} \wedge \cdots \wedge d z^{n+1}\right)=-\operatorname{trace}\left(\eta_{\beta}{ }^{\gamma}\right)=-d^{\prime} h_{\beta \bar{\alpha}} h^{\bar{\alpha} \beta}=-d^{\prime} \log \left(\operatorname{det}\left(h_{\beta \bar{\gamma}}\right)\right)$

because of (5.10). We see from (5.11) that the curvature form of the connection $-\eta$ is

$$
-d \eta=-d^{\prime \prime} d^{\prime} \log \left(\operatorname{det}\left(h_{\beta \bar{\gamma}}\right)\right) .
$$

Note that (5.12) implies that the global 2-form $-d \eta$ on $N$ is pure imaginary, that is,

$$
\operatorname{Re}(-d \eta)=0
$$

If $(N, h)$ is a Kähler manifold, the Ricci form of the Kähler manifold is given by $-2 i d \eta$ (cf. [5]) and the first Chern class $c_{1}(N)$ is represented by the Ricci form (cf. [1]). Note that the notation " $-d \eta$ " does not mean at all that it is exact.

Let $l: M \rightarrow N$ be a nondegenerate real hypersurface in $N$ and $-\eta^{*}$ the connection of $K_{M}$ induced by $-\eta$. From (5.13), we have

$$
\operatorname{Re}\left(-d \eta^{*}\right)=0
$$

where $-d \eta^{*}$ is the curvature form of the connection $-\eta^{*}$. Thus since the induced connection $-\eta^{*}$ satisfies (4.5), it determines a CR Weyl connection $D$ by virtue of Theorem 4.1. The CR Weyl connection $D$ is called the canonical $C R$ Weyl connection on $M$ induced by the Hermitian structure $h$ on $N$. By (5.5) and (5.11), the canonical CR Weyl connection $D$ satisfies (3.24). We consider when the canonical CR Weyl connection $D$ becomes CR Einstein-Weyl. Yau showed (cf. [1], [14])

Lemma 5.2. Let $N$ be a compact Kählerian manifold. If $c_{1}(N)=0, N$ admits a Kähler metric with zero Ricci form.

From this lemma, we immediately obtain 
COROLlary 5.3. Let $N$ be a compact Kählerian manifold with zero first Chern class. Then every nondegenerate real hypersurface $M$ in $N$ admits a $C R$ Einstein-Weyl structure.

Proof. From Lemma 5.2, we have a Kähler metric $h$ on $N$ with zero Ricci form. Let $-\eta$ be the connection of $K_{N}$ induced by the Hermitian connection of this metric $h$. Since the Ricci form is zero, we have $-d \eta=0$. Thus, combining this result with (5.11), we see that the connection $-\eta$ of $K_{N}$ is holomorphic. Therefore we see from Theorem 5.1 that every nondegenerate real hypersurface $M$ in $N$ admits a CR Einstein-Weyl connection. In fact the canonical CR Weyl connection induced by $h$ is CR Einstein-Weyl.

Acknowledgements. I wish to express my deep gratitude to Professor K. Sakamoto for the encouragement and suggestions which he gave me during the time I was working on this paper. I am indebted to Y. Nitta for his help.

\section{REFERENCES}

[1] A. L. Besse, Einstein manifolds, Springer-Verlag, Berlin, Heidelberg, New York, 1987.

[2] F. A. FARris, An intrinsic construction of Fefferman's CR metric, Pacific J. Math. 123 (1986), 33-45.

[ 3 ] H. JACoвowitz, The canonical bundle and realizable CR hypersurfaces, Pacific J. Math. 127 (1987), 91-101.

[4] S. Kobayashi, Differential geometry of complex vector bundles, Iwanami Shoten and Princeton University Press, 1987.

[ 5] S. Kobayashi and K. Nomizu, Foundations of differential geometry I and II, Interscience, New York, London, 1963.

[6] J. M. LeE, The Fefferman metric and pseudohermitian invariants, Trans. Amer. Math. Soc. 296 (1986), 411-429.

[ 7 ] J. M. LeE, Pseudo-Einstein structure on CR manifolds, Amer. J. Math. 110 (1988), 157-178.

[8] T. Ohкubo and K. Sakamoto, CR Einstein-Weyl structures, Tsukuba J. Math. 29 (2005), 309-361.

[9] K. SaKamoto and Y. TAKemura, On almost contact structures belonging to a CR-structure, Kodai Math. J. 3 (1980), 144-161.

[10] K. Sakamoto and Y. Takemura, Curvature invariants of CR-manifolds, Kodai Math. J. 4 (1981), 251-265.

[11] C. M. Stanton, Intrinsic connection for Levi metrics, Manuscripta Math. 75 (1992), 349-364.

[12] N. TanaKa, A differential geometric study on strongly pseudo-convex manifolds, Kinokuniya Book-Store Co. Ltd. Tokyo, 1975.

[13] S. M. Webster, Pseudo-hermitian structures on a real hypersurface, J. Differential Geom. 13 (1978), 25-41.

[14] F. ZHENG, Complex differential geometry, American Mathematical Society and International Press, 2000.

Department of Mathematics

Faculty OF SCIENCE

SAITAMA UNIVERSITY

Simo-OhKubo, Sakura-kU, Saitama 338-8570

JAPAN

E-mail: tohkubo@rimath.saitama-u.ac.jp 